$$
\begin{aligned}
& \text { Universidade de São Paulo } \\
& \text { Instituto de Física de São Carlos }
\end{aligned}
$$

Lucas Marcelo Cavalari Nardi

\title{
O modelo de Hubbard unidimensional via DFT: o potencial de troca e correlação e o funcional híbrido
}



Lucas Marcelo Cavalari Nardi

\section{O modelo de Hubbard unidimensional via DFT: o potencial de troca e correlação e o funcional híbrido}

Dissertação apresentada ao Programa de PósGraduação em Física do Instituto de Física de São Carlos da Universidade de São Paulo, para obtenção do título de mestre em Ciências.

Área de Concentração: Física Básica

Orientador: Prof. Dr. Luiz Nunes de Oliveira

Versão Corrigida

(versão original disponível na Unidade que aloja o Programa)

São Carlos 
AUTORIZO A REPRODUÇÃO E DIVULGAÇÃO TOTAL OU PARCIAL DESTE TRABALHO, POR QUALQUER MEIO CONVENCIONAL OU ELETRÔNICO PARA FINS DE ESTUDO E PESQUISA, DESDE QUE CITADA A FONTE.

Ficha catalográfica revisada pelo Serviço de Biblioteca e Informação do IFSC, com os dados fornecidos pelo(a) autor(a)

Nardi, Lucas Marcelo Cavalari

O modelo de Hubbard unidimensional via DFT: potencial de troca e correlação e o funcional híbrido / Lucas Marcelo Cavalari Nardi; orientador Luiz

Nunes de Oliveira - versão corrigida -- São Carlos, 2016

$62 \mathrm{p}$.

Dissertação (Mestrado - Programa de Pós-Graduação em Física Básica) -- Instituto de Física de São Carlos, Universidade de São Paulo, 2016.

1. Teoria do funcional da densidade. 2. Modelo de Hubbard. 3. Gap. 4. Funcional híbrido. I. Oliveira, Luiz Nunes de, orient. II. Título. 


\section{AGRADECIMENTOS}

Um trabalho como este nunca é feito sozinho. Dito isso é importante agradecer a todos que tornaram esta dissertação possível.

Em primeiro um agradecimento especial ao meu orientador Luiz Nunes de Oliveira, detendor de 13 prêmios de melhor professor, pelo conhecimento e, principalmente, pela paciência e conselhos.

À minha família pelo grande apoio e carinho. À minha namorada Gabriela pelo apoio, amor e companhia. Agradeço aos meus colegas de sala pela ajuda quando a computação ficava muito difícil, quando a física parecia obscura ou quando as regras da ABNT pareciam impossíveis de implementar no LaTex.

Aos meus colegas de graduação, pois sem a companhia deles seria difícil sobreviver à graduação.

E um agradecimento ao meu amigo Felipe pela vasta sabedoria em computação.

Agradeço também ao CNPq pelo suporte financeiro. 

Revoluções científicas são inauguradas por um sentimento crescente de que o paradigma existente cessou de funcionar adequadamente na exploração de um aspecto da natureza.

Thomas Samuel Kuhn. A estrutura das revoluções científicas. p. 84 



\section{RESUMO}

NARDI, L. M. C. O modelo de Hubbard unidimensional via DFT: o potencial de troca e correlação e o funcional híbrido. 2016. 62 p. Dissertação (Mestrado em Ciências) - Instituto de Física de São Carlos, Universidade de São Paulo, São Carlos, 2016.

A Teoria do funcional da Densidade (DFT) é muito empregada no estudo da densidade eletrônica e energia do estado fundamental de sistemas interagente de muitos elétrons. Uma de suas desvantagens é que, apesar de formalmente exata, a DFT depende de aproximações no funcional de troca e correlação $E_{x c}[n]$. Uma de suas vantagens é a possibilidade de trabalhar com a conexão adiabática, que permite conectar explicitamente o sistema interagente de interesse com uma versão não-interagente de mesma densidade. Baseado em seu escopo esta dissertação tem como objetivo tratar da DFT no modelo de Hubbard unidimensional. Uma das vantagens em trabalhar com o Hubbard é a existência de uma solução formalmente exata para a energia do modelo homogêneo e unidimensional via ansatz de Bethe, tal solução serve de base para os nossos cálculos e resultados. Outra vantagem do modelo de Hubbard é a existência de um gap que aproximações usuais na DFT falham em reproduzir. Um de nossos resultados é calcular o gap de duas formas. Uma através da derivada da solução via ansatz de Bethe e outro é tratar o Hamiltoniano pela conexão adiabática, calcular o gap pelo potencial químico, este último calculado pelo ansatz de Bethe. Ao final comparamos a precisão dos métodos, apenas para descobrir que o método via potencial químico é mais preciso. Por último usamos a conexão adiabática e a aproximação de Hartree-Fock para teorizar um funcional híbrido no modelo de Hubbard unidimensional.

Palavras-Chave: Teoria do funcional da densidade. Modelo de Hubbard. Gap. Funcional híbrido. 



\section{ABSTRACT}

NARDI, L. M. C. The one-dimensional Hubbard model via DFT: the exchange-correlation potential and the hybrid functional. 2016. 62 p. Dissertação (Mestrado em Ciências) Instituto de Física de São Carlos, Universidade de São Paulo, São Carlos, 2016.

The Density Functional Theory (DFT) is a widely used in the study of electronic density and energy of the ground-state of interacting systems consisting of many electrons. One of its disadvantages is that, although it is formally exact, the DFT depends on approximations of the exchange-correlation functional $E_{x c}[n]$. One of its advantages consists of the possibility of working with the adiabatic connection, which allows a explicit connection between the interacting system of our interest and a non-interacting system that yields the same density. Based on its scope this dissertation aims to address the DFT in the one-dimensional Hubbard model. One of the Hubbard's model advantage consists of the existence of a formally exact solution to the energy of the homogeneous one-dimensional model via Bethe ansatz, such a solution serves as the basis for our calculations and results. Anothe Hubbard's model advantage is the existence of a gap that usual approximations in DFT fail to calculate. One of our results is to calculate the gap in two different ways. One through the derivative of the Bethe ansatz solution and the other is to address the Hamiltonian through the adiabatic connection, calculate the gap through the chemical potential, the latter calculated via Bethe ansatz. In the end we compare their precisions, only to find that the one via chemical potential is more precise. At last we use the adiabatic connection and the Hartree-Fock approximation to theorize a hybrid functional in the one-dimensional Hubbard model.

KEYWORDS: Density functional theory. Hubbard model. Gap. Hybrid functional. 



\section{SUMÁRIO}

1 Introdução 13

1.1 A teoria do funcional da densidade . . . . . . . . . . . . . . . . . 13

1.2 A conexão adiabática e os funcionais híbridos . . . . . . . . . . . . . . . . . . 14

$1.3 \quad \mathrm{O}$ modelo de Hubbard . . . . . . . . . . . . . . . . . . . 15

$1.4 \quad$ A história da Teoria do Funcional da Densidade . . . . . . . . . . . . . . 16

2 A teoria do Funcional da Densidade 19

2.1 O teorema de Hohenberg e Kohn . . . . . . . . . . . . . . . . . . 19

2.2 As equações de Kohn e Sham . . . . . . . . . . . . . . . . . . . . . 22

$2.3 \quad$ A Conexão Adiabática . . . . . . . . . . . . . . . . . . . . . . . . . . . . 26

3 O modelo de Hubbard 29

3.1 O Hamiltoniano do modelo de Hubbard $1-\mathrm{D} \ldots \ldots . \ldots 29$

3.2 Exemplos paradigmáticos . . . . . . . . . . . . . . . . 33

3.2.1 Regime de interação forte . . . . . . . . . . . . . . . . . . . . 33

3.2.2 Regime de elétrons livres . . . . . . . . . . . . . . . . . . . . . 34

3.3 As simetrias do modelo de Hubbard . . . . . . . . . . . . . . . . . 36

3.3.1 Invariância translacional . . . . . . . . . . . . . . . . . . . . 36

3.3.2 Simetria partícula-buraco . . . . . . . . . . . . . . 36

3.4 A solução pelo ansatz de Bethe . . . . . . . . . . . . . . . . . . 38

4 Resultados $\quad 41$

4.1 A conexão adiabática no modelo de Hubbard . . . . . . . . . . . . . . . . 41

$4.2 \quad$ O potencial químico . . . . . . . . . . . . . . . . . . . . . . . . 43

$4.3 \quad \mathrm{O}$ potencial de troca e correlação . . . . . . . . . . . . . . . . 44 
4.3 .1 O problema da derivada . . . . . . . . . . . . . . . . . . . . 46

4.4 Derivação alternativa do potencial de troca e correlação . . . . . . . . . . . 48

4.5 O modelo de Hubbard $1-\mathrm{D} \ldots \ldots \ldots$. . . . . . . . . . . . . . . . . 49

$4.6 \quad$ Funcionais Híbridos . . . . . . . . . . . . . . . . . . . . . . . . . . . . . 51

4.6.1 A aproximação de Hartree-Fock . . . . . . . . . . . . . . . . . . . . . . . 53

4.6.2 O funcional híbrido para o modelo de Hubbard 1-D . . . . . . . . . . . . . 54

5 Considerações Finais 59

$\begin{array}{ll}\text { REFERÊNCIAS } & 61\end{array}$ 


\section{Introdução}

A Teoria do Funcional da Densidade (DFT) já tem mais de cinco décadas. Os dois trabalhos que a sustentam, de Hohenberg e Kohn (1) e de Kohn e Sham (2), já definiram o objetivo do formalismo: determinar a densidade eletrônica e a energia do estado fundamental de gases de elétrons não-homogêneos. Exemplos de tais gases são encontrados em átomos, moléculas e em sólidos, sistemas que, exceto nos casos de átomos ou de moléculas com poucos elétrons, definem problemas complexos. A complexidade vem da interação elétron-elétron, que exige tratar simultaneamente todos as partículas, e do potencial atrativo dos núcleos, que reduz a simetria. A DFT simplifica o tratamento porque permite trabalhar com a densidade, em lugar da função de onda do sistema de interesse e porque mostra que a densidade e a energia do estado fundamental do sistema interagente podem ser obtidos a partir de um sistema com número igual de elétrons que não interagem.

\subsection{A teoria do funcional da densidade}

Os dois pilares teóricos da DFT são:

\section{O trabalho de Hohenberg e Kohn:}

O teorema de Hohenberg e Kohn mostra que a densidade e energia do estado fundamental de um sistema podem ser determinados univocamente tendo um ansatz inicial para a densidade eletrônica do estado fundamental do sistema. Graças ao primeiro teorema podemos utilizar funcionais da densidade $n(\vec{r})$ para estudar o estado fundamental de um sistema não degenerado, tendo assim uma relação biunívoca entre energia e densidade do estado fundamental.

Em 1964, quando o trabalho de Hohenberg e Kohn foi publicado, o preço da simplificação parecia elevado. Para associar o sistema não-interagente ao sistema interagente é necessário conhecer um funcional da densidade eletrônica sobre o qual se tem, a princípio, pouca informação. Embora tenha mostrado que a energia do estado fundamental é um funcional $E[n]$ 
da densidade $n(\vec{r})$ do sistema, e discutir corolários de seu teorema, o trabalho de Hohenberg e Kohn não ofereceu um método para se encontrar $E[n]$.

O trabalho de Kohn e Sham:

O artigo de Kohn e Sham propôs um procedimento aproximado para se encontrar o funcional. Para isso, os autores definiram um novo funcional $E_{x c}[n]$, conhecido como funcional de troca e correlação, como a diferença entre o funcional $E[n]$ e a soma de:

(i) a energia cinética do gás não-interagente;

(ii) a energia potencial dos elétrons no potencial externo que quebra a uniformidade do gás;

(iii) a energia eletrostática clássica associada à densidade $n(r)$.

Em outras palavras, eles dividiram o potencial $E[n]$ em uma parte relativamente grande que pode ser facilmente computada [a soma das energias (i), (ii) e (iii)] e outra parte, pequena e desconhecida $\left(E_{x c}[n]\right)$.

É muito dificíl, entretanto, desenvolver técnicas de aproximação para $E_{x c}$. Por isso, para descrever $E_{x c}[n]$, Kohn e Sham propuseram a aproximação da densidade local (LDA). Em linhas gerais, a LDA consiste em:

(i) dividir o gás em um número muito grande de pequeno blocos, de forma que a densidade eletrônica dentro de cada bloco seja aproximadamente constante;

(ii) supor que $E_{x c}[n]$ é simplesmente a soma das contribuições de cada bloco;

(iii) calcular a contribuição de cada bloco como se ele estivesse em um gás homogêneo com densidade eletrônica igual à densidade média dentro do boco.

Boa parte do sucesso da DFT se deve ao surpreendente desempenho da LDA, que motivou outros trabalhos e ofereceu pistas que guiaram o desenvolvimento de aproximações mais precisas para o funcional $E_{x c}[n]$. (3) Outro guia importante para gerar novas formas aproximadas para $E_{x c}[n]$ é o resultado matemático conhecido como conexão adiabática.

\subsection{A conexão adiabática e os funcionais híbridos}

A conexão adiabática oferece uma expressão matemática formalmente exata para o funcional. Embora envolva operações ainda mais difíceis de efetuar do que a expansão de $E_{x c}[n]$, 
a conexão adiabática oferece alternativas para tratamento aproximado. A partir dela, é fácil argumentar que, aproximadamente, a contribuição da interação elétron-elétron para o funcional é a combinação linear entre a energia de um gás fracamente interagente com densidade $n(\vec{r})$ e a energia de um gás fortemente interagente com a mesma densidade. A primeira é bem descrita pelo tratamento de Hartree e Fock, e assim dispensa a LDA. A segunda precisa ser descrita pela aproximação local. As aproximações para o funcional $E_{x c}[n]$ resultantes da combinação são conhecidas como funcionais híbridos.

Os coeficientes da combinação que definem os funcionais híbridos são desconhecidos. Embora sejam associados a uma igualdade matemática exata e embora possam ser estimados com argumentos heurísticos, eles são frequentemente encarados como parâmetros ajustáveis, que devem ser otimizados para reproduzir resultados experimentais. Esta dissertação descreve um estudo do modelo de Hubbard unidimensional que alimenta a discussão de critérios para determinar tais coeficientes. O modelo de Hubbard unidimensional é um campo de testes interessante porque a energia do estado fundamental do modelo uniforme é conhecida.

\subsection{O modelo de Hubbard}

O modelo de Hubbard é uma aproximação muito utilizada na física do estado sólido para estudar férmions. Esse modelo é considerado uma boa aproximação para partículas em um potencial periódico com temperaturas suficientemente baixas, desde que os elétrons ocupem a banda de menor energia e que interações de longo alcance possam ser ignoradas. O modelo de Hubbard pode ser escrito pelo seguinte Hamiltoniano:

$$
\hat{H}=-t \sum_{j=1}^{2 L} \sum_{a=\uparrow, \downarrow}\left(c_{j, a}^{\dagger} c_{j+1, a}+\text { h.c. }\right)+U \sum_{j=1}^{2 L} n_{j, \uparrow} n_{j, \downarrow}-\mu \sum_{j} c_{j}^{\dagger} c_{j} .
$$

Um dos grandes trunfos desse modelo é prever a existência de gaps na transição de Mott, que pode ser entendido como uma mudança na estrutura com a adição de poucos elétrons.

A expressão para a energia do estado fundamental do modelo de Hubbard uniforme serviu de base para aproximações de densidade local para o funcional da densidade do modelo, conhecida pela sigla $B A-L D A$. A BA-LDA exibe qualidades não encontradas na LDA tradicional. Por exemplo, ela reproduz, aproximadamente, o gap no espectro de excitação do modelo quando a ocupação média é igual a um elétron por sítio. De fato, com uma simples redefinição de procedimentos, a BA-LDA consegue calcular exatamente o gap, um primeiro resultado desta 
dissetação.

O foco do trabalho, entretanto, é a contrução de um funcional híbrido para o modelo de Hubbard. As operações matemáticas que definem a conexão adiabática se reduzem a uma integral simples, a qual pode ser computada numericamente. A participação relativa dos gases fraca e fortemente interagentes pode portanto ser determinada e discutida em função dos parâmetros do modelo. Os resultados poderão ajudar na construção de funcionais híbridos para os modelos de Hubbard 2-D e 3-D e para o gás de elétrons 3-D.

\subsection{A história da Teoria do Funcional da Densidade}

A quantidade de pesquisa que utiliza ou procura aprimorar a DFT hoje é muito grande. Até a década de 90, porém, o formalismo atraiu muito pouca atenção. A Fig. 1.1 mostra a diferença na quantidade de trabalhos e artigos, consequência de grandes avanços na história dessa teoria.

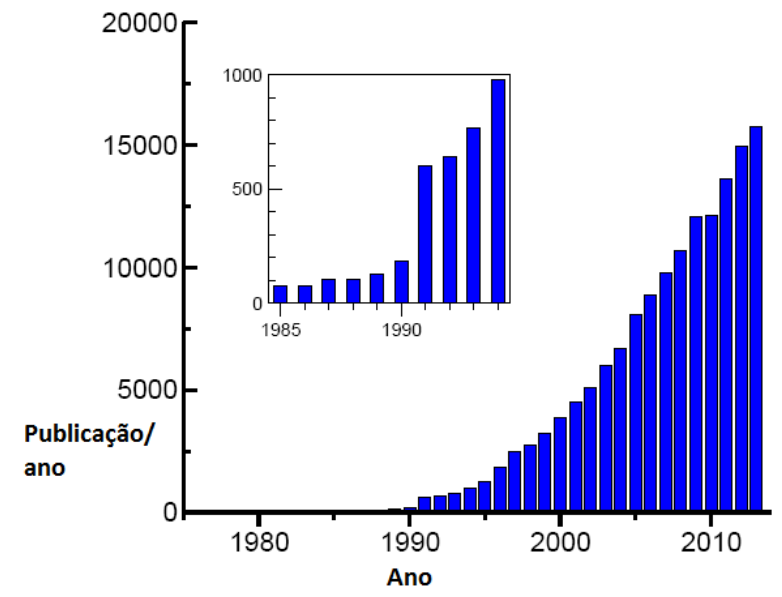

Figura 1.1 - Número de publicações por ano entre 1975-2013 nos tópicos density functional e DFT, de acordo com o Web of Knowledge (Julho 2014). A inserção mostra, em escala expandida, os anos próximos de 1990.

Fonte: JONES. (4) 
Na década de 60 apareceram os já mencionados trabalhos fundamentas de Hohenberg e Kohn e de Kohn e Sham. Dez anos mais tarde, a conexão adiabática foi construída e serviu para explicar a precisão dos resultados produzidos pela LDA. O trabalho de Gunnarsson e Lundqvist $(5,6)$ também introduziu a teoria do funcional da densidade de spin e estendeu a aproximação da densidade local para gases magneticamente polarizados, o que expandiu o escopo da teoria para além dos sistemas diamagnéticos. Abriu-se, dessa forma, a porta para o tratamento da interação elétron-elétron em sólidos, sistemas para os quais a LDA logo se mostrou adequada, pois, em comparação com átomos ou moléculas, a densidade varia lentamente. Data dessa época a percepção de que a DFT é inadequada para calcular gaps de semicondutores. Algum tempo depois ficou claro que a dificuldade se deve à LDA, que não consegue reproduzir a descontinuidade pela qual a derivada do funcional da densidade passa quando a energia chega ao topo de uma banda cheia. (7)

As primeiras tentativas de aprimoramento da LDA buscaram expressar o funcional da densidade em função da densidade e seu gradiente, sem nenhum sucesso. O progresso só veio com a melhor compreensão das condições que a LDA satisfaz e que a tornam relativamente precisa. A busca por aproximações mais precisas para o funcional da densidade se concentrou, a partir de então, em expressões dependentes de $n(\vec{r})$ e $\nabla n(\vec{r})$ que respeitem certas regras de soma, (3) conhecidas como aproximações de gradiente generalizadas (GGA).

No início dos anos 90, foi introduzido o conceito de funcional híbrido. (8) Para ir além da GGA, os funcionais híbridos incorporam uma parcela proporcional à energia de Hartree e Fock para a densidade em questão. Essa parcela é não local, isto é, não depende apenas da densidade local ou do seu gradiente. Isso tem um custo computacional relativamente elevado, mas em troca oferece precisão. A principal limitação dos funcionais híbridos é a indefinição a priori dos coeficientes da combinação. Ajustada adequadamente a combinação, os híbridos conseguem reproduzir, por exemplo, os gaps experimentais em semicondutores.

Como explicado anteriormente, este trabalho procura entender melhor os funcionais híbridos com base em aplicação ao modelo de Hubbard 1-D. Essa representação de um gás uniforme é interessante porque a solução exata (9) e estudos subsequentes ofereceram visão abrangente de suas propriedades. Uma outra vantagem do modelo de Hubbard é a solução conhecida para qualquer valor do parâmetro $U$, o parâmetro de interação entre os elétrons. Graças a isso, a discussão que será apresentada no capítulo 4 pôde focalizar os regimes de interação fraca e de interação forte, bem como a transição entre eles. As mudanças no funcional híbrido à medida que $U$ cresce constituem o aspecto mais ilustrativo dos resultados. Antes de chegar a eles, porém, convém discutir, com mais detalhe a DFT e o modelo de Hubbard. 


\section{A teoria do Funcional da Densidade}

A Teoria do Funcional da Densidade oferece uma solução relativamente simples e potencialmente muito eficiente para o cálculo da energia do estado fundamental de um gás não-homogêneo de elétrons interagentes. Ela é eficiente porque transforma um problema que envolve uma função de $3 N$ variáveis (a função de onda) em outro que trabalha com uma função de apenas 3 variáveis (a densidade). Na prática, o método depende de aproximações, mas ele é formalmente exato, uma consequência das sólidas bases que o sustentam: o Teorema de Hohenberg-Kohn (1) e as equações de Kohn-Sham. (2)

\subsection{O teorema de Hohenberg e Kohn}

O teorema de Hohenberg-Kohn garante que, em um sistema sem degenerescências, finito e com $N$ elétrons interagentes por meio de um dado potencial elétron-elétron, existe uma correspondência bijetora entre o potencial externo $\nu(\vec{r})$ e a densidade eletrônica do estado fundamental, $n_{0}(\vec{r})$. O teorema parte de algumas definições.

Em uma região finita do espaço, tem-se um sistema de $N$ elétrons interagentes descritos pelo Hamiltoniano

$$
\hat{H}=\hat{T}+\hat{V}+\hat{W}
$$

onde os termos cinético, potencial e interagente são

$$
\begin{gathered}
\hat{T}=\sum_{j=1}^{N}-\frac{\hbar^{2}}{2 m} \vec{\nabla}_{j}^{2}, \\
\hat{V}=\sum_{j=1}^{N} \nu\left(\vec{r}_{j}\right)
\end{gathered}
$$


e

$$
\hat{W}=\frac{1}{2} \sum_{j, k, j \neq k}^{N} \omega\left(\left|\vec{r}_{j}-\vec{r}_{k}\right|\right),
$$

respectivamente.

A energia do estado fundamental $|\psi\rangle$ de $\hat{H}$ é dada pela equação

$$
E_{0}=\langle\psi|\hat{H}| \psi\rangle
$$

Dada a interação $\hat{W}$, o Hamiltoniano depende apenas do potencial $\hat{V}$. Conhecido $\hat{V}$ é em princípio possível diagonalizar $\hat{H}$ e encontrar o estado fundamental $|\psi\rangle$. Em seguida, será possível calcular a densidade $n(\vec{r})$ associada ao estado fundamental,

$$
n(\vec{r})=\langle\psi|\hat{n}| \psi\rangle
$$

onde $\hat{n}$ é o operador densidade.

A cada potencial externo $\hat{V}$ é portanto possível associar uma (única) densidade $n(\vec{r})$. O teorema quer mostrar que a cada densidade $n(\vec{r})$ corresponde um (único a menos de uma constante arbitrária) potencial externo.

Teorema (2.1.1): Em um sistema não degenerado, há uma relação bijetora entre o potencial externo, $\nu\left(\vec{r}_{j}\right)$ e a densidade eletrônica do estado fundamental, $n_{0}(\vec{r})$.

Dedução: O raciocínio se apoia em uma demonstração por absurdo. Suponhamos que duas funções de onda distintas por mais do que uma transformação de gauge global sejam auto-estados fundamentais de dois Hamiltonianos distintos

$$
\hat{H}=\hat{T}+\hat{V}+\hat{W}
$$

e

$$
\hat{H}^{\prime}=\hat{T}+\hat{V}^{\prime}+\hat{W}
$$

onde os potenciais $\hat{V}$ e $\hat{V}^{\prime}$ diferem por mais do que uma constante, e suponhamos que os auto-estados fundamentais $|\psi\rangle$ de $\hat{H}$ e $\left|\psi^{\prime}\right\rangle$ de $\hat{H}^{\prime}$ gerem a mesma densidade $n(\vec{r})$.

Sejam $E_{0}$ e $E_{0}^{\prime}$ as energias dos estados fundamentais de $\hat{H}$ e $\hat{H}^{\prime}$, respectivamente. Segundo o princípio variacional de Rayleigh-Ritz

$$
E_{0}<\left\langle\psi^{\prime}|\hat{H}| \psi^{\prime}\right\rangle,
$$


com desigualdade estrita, já que $\left|\psi^{\prime}\right\rangle$ não é o auto-estado fundamental de $\hat{H}$.

As equações (2.1.7) e (2.1.8) permitem reescrever a desigualdade (2.1.9) na forma

$$
E_{0}<\left\langle\psi^{\prime}\left|\hat{H}^{\prime}\right| \psi^{\prime}\right\rangle+\left\langle\psi^{\prime}\left|\hat{V}-\hat{V}^{\prime}\right| \psi^{\prime}\right\rangle
$$

ou seja, na forma

$$
E_{0}<\left\langle\psi^{\prime}\left|\hat{H}^{\prime}\right| \psi^{\prime}\right\rangle+\int\left(\nu(\vec{r})-\nu^{\prime}(\vec{r})\right) n(\vec{r}) \mathrm{d}^{3} r
$$

que equivale a

$$
E_{0}<E_{0}^{\prime}+\int\left(\nu(\vec{r})-\nu^{\prime}(\vec{r})\right) n(\vec{r}) \mathrm{d}^{3} r
$$

Podemos agora refazer o raciocínio a partir do auto-estado de $\hat{H}$, isto é, partir da desigualdade

$$
E_{0}^{\prime}<\left\langle\psi\left|\hat{H}^{\prime}\right| \psi\right\rangle
$$

para concluir que

$$
E_{0}<E_{0}^{\prime}+\int\left(\nu^{\prime}(\vec{r})-\nu(\vec{r})\right) n(\vec{r}) \mathrm{d}^{3} r
$$

já que as densidades de $|\psi\rangle$ e $\left|\psi^{\prime}\right\rangle$ são iguais a $n(\vec{r})$.

Basta agora adicionar membro a membro as desigualdades (2.1.13) e (2.1.14) para chegarmos ao absurdo $E_{0}+E_{0}^{\prime}<E_{0}^{\prime}+E_{0}$. Segue que as densidades dos auto-estados $|\psi\rangle \mathrm{e}$ $\left|\psi^{\prime}\right\rangle$ são necessariamente distintas e que a relação entre o potencial $\nu(r)$ e a densidade $n(\vec{r})$ é bijetora.

Assim como o potencial, a densidade eletrônica determina todas as propriedades físicas de um sistema de $N$ elétrons interagentes. Para encontrar as propriedades do estado fundamental de um sistema de $N$ elétrons, é desnecessário encontrar a sua função de onda, que depende de $3 N$ variáveis. Basta encontrar a densidade, que depende das três coordenadas espaciais. Isso em tese, porque até aqui dependemos da função de onda para encontrar a densidade.

Hohenberg e Kohn mostraram um caminho direcionado para o cálculo direto da densidade. (1) Suponhamos dada uma densidade $\bar{n}(\vec{r})$. De acordo com o Teorema de Hohenberg-Kohn, ela está associada a um único estado fundamental $|\bar{\psi}\rangle$. Se esse estado for conhecido, dado o Hamiltoniano (2.1.1), podemos calcular o valor médio esperado

$$
\bar{E}=\langle\bar{\psi}|\hat{T}+\hat{V}+\hat{W}| \bar{\psi}\rangle
$$


O procedimento resumido pela equação (2.1.15) define um funcional $E[\bar{n}]$ da densidade $\bar{n}(\vec{r})$, uma vez que, dada uma função $\bar{n}$, a construção acima descrita fornece o número $\bar{E}$. Podemos portanto reescrever a equação (2.1.15) na forma

$$
E[\bar{n}]=\langle\bar{\psi}|\hat{T}+\hat{V}+\hat{W}| \bar{\psi}\rangle
$$

Nessa notação, o princípio variacional de Rayleigh-Ritz garante que

$$
E[n]<E[\bar{n}] \quad(\bar{n} \neq n)
$$

onde $n(\vec{r})$ é a densidade do estado fundamental de $\hat{H}$.

A desigualdade (2.1.17) expressa o raciocínio de Hohenberg e Kohn. A densidade do estado fundamental minimiza o funcional $E[n]$. Para explorar essa noção, porém, precisamos conhecer o funcional e precisamos de um método para encontrar a função que o minimiza. Esse é o assunto da próxima seção.

\subsection{As equações de Kohn e Sham}

Kohn e Sham definiram um gás de elétrons não-interagentes, conhecido como sistema de Kohn-Sham, sujeito a um potencial $\hat{V}_{s}$ tal que a densidade do seu estado fundamental seja idêntica a do sistema interagente com Hamiltoniano (2.1.1). O sistema de Kohn-Sham tem o Hamiltoniano

$$
\hat{H}_{s}=\hat{T}+\hat{V}_{s}
$$

com a energia cinética definida na equação (2.1.2).

Para encontrar o potencial $\hat{V}_{s}$, os autores definiram o funcional de troca e correlação, $E_{x c}[n]$ pela expressão

$$
E[n] \equiv T_{s}[n]+\int \nu(\vec{r}) n(\vec{r}) \mathrm{d}^{3} r+\frac{e^{2}}{2} \int \frac{n(\vec{r}) n\left(\overrightarrow{r^{\prime}}\right)}{\left|\vec{r}-\overrightarrow{r^{\prime}}\right|} \mathrm{d}^{3} r \mathrm{~d}^{3} r^{\prime}+E_{x c}[n],
$$

onde

$$
T_{s}[n]=\left\langle\psi_{s}|\hat{T}| \psi_{s}\right\rangle
$$

é o valor médio esperado da energia cinética no estado fundamental $\left|\psi_{s}\right\rangle$ do gás não intera- 
gente. No lado direito da equação (2.2.2) o segundo termo é a energia de Hartree, isto é, a energia de Coulomb clássica associada à densidade $n(r)$, e a última parcela é o funcional de troca e correlação.

Da mesma forma que a energia do sistema interagente, a energia do estado fundamental do sistema não interagente é um funcional da densidade, que tem forma análoga à da equação (2.1.16):

$$
E_{s}[n]=T_{s}[n]+\int n(\vec{r}) \nu_{s}(\vec{r}) \mathrm{d}^{3} r
$$

onde $\nu_{s}(\vec{r})$ é o potencial que define o operador $\hat{V}_{s}$.

A densidade $n(\vec{r})$ minimiza tanto $E[n]$ como $E_{s}[n]$. A uma mesma variação infinitesimal da densidade $\delta n(\vec{r})$ correspondem as condições $\delta E[n]=0$ e $\delta E_{s}[n]=0$. Da equação (2.2.2) temos, portanto, que

$$
0=\frac{\delta T_{s}[n]}{\delta n}+\nu(\vec{r})+e^{2} \int \frac{n\left(\overrightarrow{r^{\prime}}\right)}{\left|\vec{r}-\overrightarrow{r^{\prime}}\right|} \mathrm{d}^{3} r^{\prime}+\frac{\delta E_{x c}[n]}{\delta n},
$$

e da equação (2.2.3), que

$$
0=\frac{\delta T_{s}[n]}{\delta n}+\nu_{s}(\vec{r})
$$

Subtraímos então, membro a membro, a equação (2.2.6) da equação (2.2.5) para eliminar o termo da energia cinética que aparece nas duas igualdades. Resulta que

$$
\nu_{s}(\vec{r})=\int \nu(\vec{r}) n(\vec{r}) \mathrm{d}^{3} r+e^{2} \int \frac{n\left(\overrightarrow{r^{\prime}}\right)}{\left|\vec{r}-\overrightarrow{r^{\prime}}\right|} \mathrm{d}^{3} r^{\prime}+v_{x c}[n](\vec{r}) .
$$

O último termo à direita é o potencial de troca e correlação

$$
\nu_{x c}[n](\vec{r}) \equiv \frac{\delta E_{x c}[n](\vec{r})}{\delta n} .
$$

Se o potencial $\nu_{s}(\vec{r})$ for encontrado, o Hamiltoniano do gás não interagente poderá ser diagonalizado numericamente e tanto a densidade como a energia do estado fundamental do sistema de Kohn-Sham poderão ser imediatamente determinados. Faltará ainda relacionar essa energia com a do sistema interagente, mas a densidade é a mesma para os dois sistemas. A equação (2.2.8) relaciona o potencial com a própria densidade e com o potencial de troca e correlação, que por sua vez é dado pela derivada funcional de $E_{x c}[n]$ na equação (2.2.8).

O funcional de troca e correlação não é conhecido a priori, dado que sua definição, na equação (2.2.2), depende do funcional $E[n]$, que também é desconhecido. Na prática, como 
vemos, será necessária uma aproximação para $E_{x c}[n]$, que precisa vir de cálculos independentes da DFT. Veremos adiante como isso pode ser feito.

Mesmo que $v_{x c}[n]$ seja conhecido, por outro lado, o cálculo do potencial $\nu_{s}$ depende de conhecermos a densidade, isto é, do próprio resultado que desejamos. Esse aparente impasse é resolvido por um ciclo auto-consistente, que começa com uma escolha educada para a densidade $n(\vec{r})$. A Fig. (2.1) descreve, esquematicamente, as etapas que compõem o ciclo.

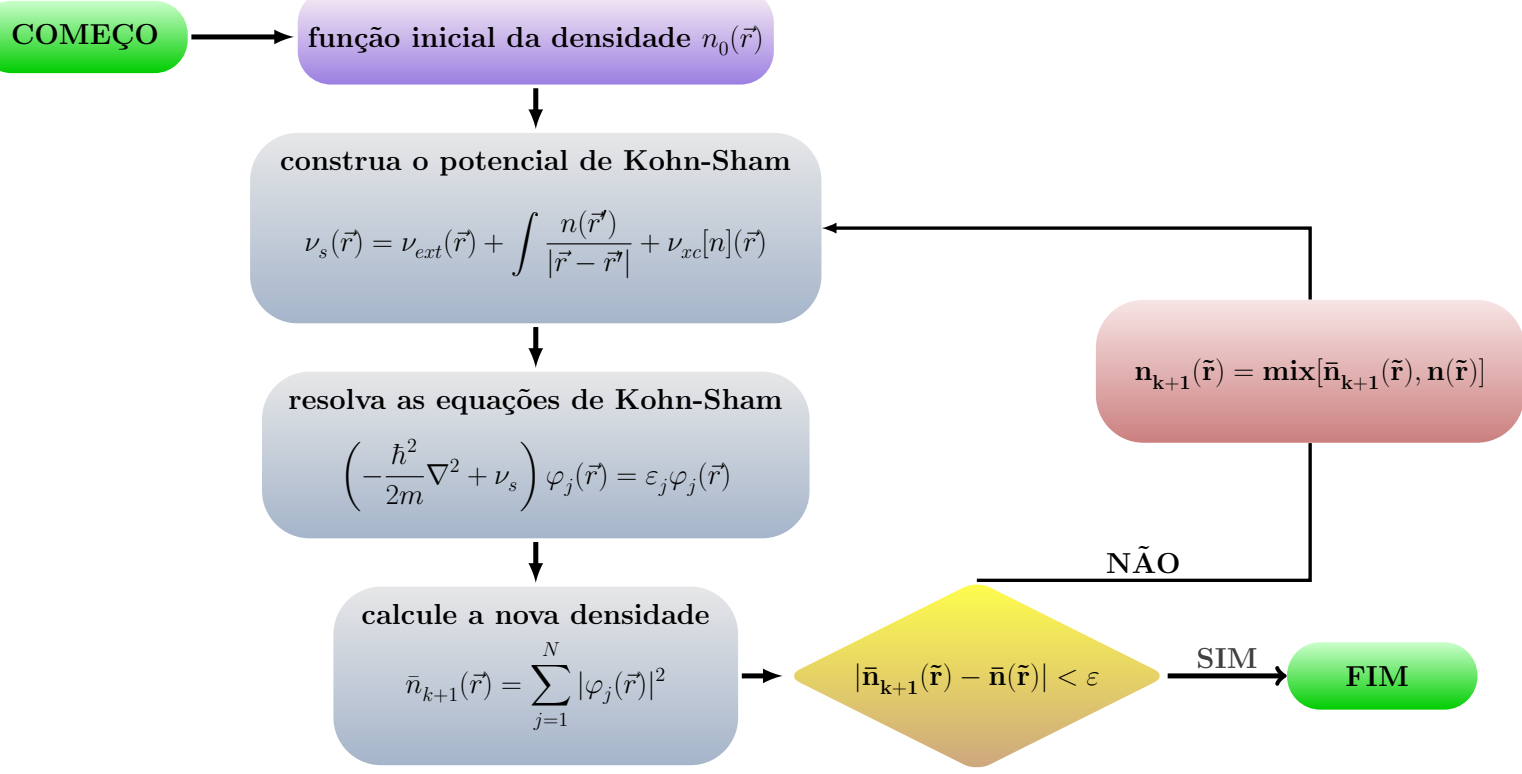

Figura 2.1 - Ciclo auto-consistente de Kohn e Sham.

Fonte: Elaborada pelo autor. 
São produtos do ciclo as energias de Kohn-Sham $\epsilon_{j}(j=1,2, \ldots, N)$ e a densidade $n(\vec{r})$. De posse dos autovalores e da densidade, podemos calcular $E[n]$. Para isso, primeiro notamos que a energia cinética do gás não-interagente pode ser escrita na forma

$$
T_{s}[n]=\sum_{j=1}^{N} \varepsilon_{j}-\int n(\vec{r}) \nu_{s}[n](\vec{r}) \mathrm{d}^{3} r .
$$

Substituímos a expressão (2.2.7) para $\nu_{s}$ no lado direito da equação (2.2.9) e substituímos o resultado para $T_{s}[n]$ no lado direito da identidade (2.2.2) para obter uma igualdade mais simples para a energia do estado fundamental:

$$
E[n]=\sum_{j=1}^{N} \varepsilon_{j}-\frac{1}{2} \int d^{3} r \int d^{3} r^{\prime} \frac{n(\vec{r}) n\left(\vec{r}^{\prime}\right)}{\left|\vec{r}-\vec{r}^{\prime}\right|}-\int n(\vec{r}) \nu_{x c}[n](\vec{r}) \mathrm{d}^{3} r+E_{x c}[n] .
$$

Dada uma aproximação para o funcional $E_{x c}[n]$, o procedimento auto-consistente definido pelo ciclo da Fig. (2.1) permite determinar, portanto, a densidade $n(r)$ e a energia fundamental $E[n]$. Comparado com o método de Thomas-Fermi (10), que também emprega a densidade como variável fundamental, o procedimento de Kohn-Sham é superior porque trata exatamente o funcional da energia cinética.

Ele depende, entretanto, de uma aproximação para $E_{x c}$. A mais simples é a aproximação de densidade local (LDA). Esta supõe que a densidade de energia de troca e correlação em um infinitésimo de volume na posição $\vec{r}$ é dada pela densidade de energia de troca e correlação de um outro sistema paralelo, chamado de sistema referencial, onde a densidade eletrônica é constante. Embora o gás homogêneo de elétrons interagentes seja normalmente o sistema referencial (3), é possível construir a LDA a partir de outros sistemas, como pode ser visto na Ref. (11)

O procedimento de Kohn-Sham relaciona o sistema interagente com um sistema nãointeragente de igual densidade. Ele não é, porém, a única conexão entre os dois sistemas, e nem mesmo a mais simples, conforme veremos a seguir. 


\subsection{A Conexão Adiabática}

Para relacionar a energia do estado fundamental do sistema interagente com a do gás de Kohn-Sham, mostra-se conveniente uma extensão da equação (2.1.1), dada pelo Hamiltoniano

$$
\hat{H}_{\lambda}=\hat{T}+\lambda \hat{W}+\hat{V}_{\lambda}
$$

onde $\lambda$ é um parâmetro adimensional arbitrário no intervalo $0 \leq \lambda \leq 1$, e o potencial $\hat{V}_{\lambda}$ é escolhido de forma que o estado fundamenta $\left|\psi_{\lambda}\right\rangle$ de $H_{\lambda}$ tenha a densidade $n(r)$ do auto-estado fundamental do Hamiltoniano (2.1.1):

$$
\left\langle\psi_{\lambda}|\hat{n}| \psi_{\lambda}\right\rangle=\left\langle\psi_{\lambda}|\hat{n}| \psi_{\lambda}\right\rangle=n(\vec{r}) \quad(0 \leq \lambda \leq 1)
$$

Em particular, com $\lambda=0$ o potencial $\nu_{\lambda}$ coincide com $\nu_{s}$ e o Hamiltoniano $H_{\lambda}$ coincide com o da equação (2.2.1), de Kohn-Sham. Para $\lambda=1$, o potencial $\nu_{\lambda}$ coincide com o externo, e $H_{\lambda}$ coincide com o Hamiltoniano interagente, da equação (2.1.1). À medida que $\lambda$ cresce de zero à unidade, o Hamiltoniano $H_{\lambda}$ evolui desde o sistema de Kohn-Sham até o sistema de interesse.

Para cada valor de $\lambda$, pode-se, em princípio, calcular a energia do estado fundamental

$$
E_{\lambda}[n] \equiv\left\langle\psi_{\lambda}\left|H_{\lambda}\right| \psi_{\lambda}\right\rangle
$$

que obedece à seguinte equação diferencial, ditada pelo Teorema de Hellmann-Feynman:

$$
\frac{d E_{\lambda}}{d \lambda}=\left\langle\psi_{\lambda}\left|\frac{\partial H_{\lambda}}{\partial \lambda}\right| \psi_{\lambda}\right\rangle
$$

A derivada parcial no elemento de matriz à direita na equação (2.3.4) pode ser obtida da equação (2.3.1):

$$
\frac{\partial H_{\lambda}}{\partial \lambda}=\hat{W}+\frac{d \hat{V}_{\lambda}}{d \lambda}
$$

Assim, da equação (2.3.4) segue imediatamente que

$$
E_{\lambda=1}-E_{\lambda=0}=\int_{0}^{1}\left\langle\psi_{\lambda}|\hat{W}| \psi_{\lambda}\right\rangle \mathrm{d} \lambda+\int_{0}^{1}\left\langle\psi_{\lambda}\left|\frac{d \hat{V}_{\lambda}}{d \lambda}\right| \psi_{\lambda}\right\rangle \mathrm{d} \lambda
$$

O primeiro termo à esquerda na equação (2.3.6) é a energia $E[n]$ do estado fundamental do Hamiltoniano interagente, enquanto o segundo termo é a energia $E_{s}[n]$ do Hamiltoniano 
de Kohn-Sham. O último termo à direita pode ser escrito na forma mais explícita

$$
\left\langle\psi_{\lambda}\left|\frac{d \hat{V}_{\lambda}}{d \lambda}\right| \psi_{\lambda}\right\rangle=\int n(\vec{r}) \frac{d \nu_{\lambda}}{d \lambda} \mathrm{d}^{3} r
$$

ou seja,

$$
\left\langle\psi_{\lambda}\left|\frac{d \hat{V}_{\lambda}}{d \lambda}\right| \psi_{\lambda}\right\rangle=\frac{d}{d \lambda} \int n(\vec{r}) \nu_{\lambda} \mathrm{d}^{3} r
$$

Concluímos que

$$
\int_{0}^{1}\left\langle\psi_{\lambda}\left|\frac{d \hat{V}_{\lambda}}{d \lambda}\right| \psi_{\lambda}\right\rangle \mathrm{d} \lambda=\int n(\vec{r}) \nu_{1}(\vec{r}) \mathrm{d}^{3} r-\int n(\vec{r}) \nu_{0}(\vec{r}) \mathrm{d}^{3} r
$$

ou, dado que $\nu_{1}$ é o potencial externo $\nu$ e que $\nu_{0}$ é o potencial de Kohn-Sham $\nu_{s}$,

$$
\int_{0}^{1}\left\langle\psi_{\lambda}\left|\frac{d \hat{V}_{\lambda}}{d \lambda}\right| \psi_{\lambda}\right\rangle \mathrm{d} \lambda=\int n(\vec{r}) \nu(\vec{r}) \mathrm{d}^{3} r-\int n(\vec{r}) \nu_{s}(\vec{r}) \mathrm{d}^{3} r .
$$

Podemos agora substituir o lado direito da equação (2.3.10) no lugar da última integral à direita da equação (2.3.6), para obter a igualdade

$$
E[n]=E_{s}[n]-\int n(\vec{r}) \nu_{s}(\vec{r}) \mathrm{d}^{3} r+\int n(\vec{r}) \nu(\vec{r}) \mathrm{d}^{3} r+\int_{0}^{1}\left\langle\psi_{\lambda}|\hat{W}| \psi_{\lambda}\right\rangle \mathrm{d} \lambda .
$$

A combinação dos dois primeiros termos à direita na equação (2.3.11), isto é, a diferença entre a energia total do gás de Kohn-Sham e a energia potencial do mesmo gás nada mais é do que a energia cinética $T_{s}[n]$. Temos, assim, que

$$
E[n]=T_{s}[n]+\int n(\vec{r}) \nu(\vec{r}) \mathrm{d}^{3} r+\int_{0}^{1}\left\langle\psi_{\lambda}|\hat{W}| \psi_{\lambda}\right\rangle \mathrm{d} \lambda .
$$

resultado conhecido como a conexão adiabática, porque na integração sobre $\lambda$ o estado fundamental $\left|\psi_{\lambda}\right\rangle$ se adapta progressivamente às mudanças na interação $\lambda \hat{W}$, que transformam o Hamiltoniano de Kohn-Sham no Hamiltoniano do gás interagente.

Podemos comparar a conexão adiabática com a definição do funcional de troca e correlação, na equação (2.2.2). A comparação mostra imediatamente que

$$
E_{x c}[n]=\int_{0}^{1}\left\langle\psi_{\lambda}|\hat{W}| \psi_{\lambda}\right\rangle \mathrm{d} \lambda-E_{H}[n],
$$

onde $E_{H}[n]$ é a energia de Hartree.

Embora exata, essa expressão é pouco prática para computação do funcional de troca e correlação, porque calcular o integrando no primeiro termo à direita é tão complexo quanto 
calcular a energia do estado fundamental do gás interagente. A equação (2.3.13) oferece uma visão diferente que tem guiado a construção de aproximações para $E_{x c}[n]$ desde a descoberta da conexão adiabática, na década de 1970. (12) Em particular, ela oferece um ponto de partida para a construção de funcionais híbridos, conforme será discutido no Capítulo 4. 


\section{O modelo de Hubbard}

O modelo de Hubbard foi introduzido na década de 1960 pelo físico britânico John Hubbard, com o objetivo de descrever os graus de liberdade eletrônicos dos sólidos. Em poucas palavras, o modelo descreve a competição entre a energia cinética dos elétrons, que tende a distribuir as funções de onda, e a interação elétron-elétron, que tende a localizá-las. A transição de Mott, em que um sistema eletrônico passa de um regime em que a energia cinética predomina a outro em que interação é a força dominante e assim deixa abruptamente de conduzir eletricidade, está no seu escopo. Nas últimas décadas, o modelo tem atraído muito interesse porque alguns elementos que parecem responsáveis pelo comportamento dos materiais semicondutores de alta temperaturas críticas são encontrados no Hamiltoniano do modelo. Neste capítulo, faremos uma recapitulação da sua derivação, de algumas de suas propriedades e da solução exata da versão unidimensional.

\subsection{O Hamiltoniano do modelo de Hubbard 1-D}

Um sólido consiste de íons e elétrons condensados em uma estrutura cristalina tridimensional. Na aproximação de Born e Oppenheimer, estritamente válida no limite em que a energia cinética dos íons é muito menor do que a dos elétrons, os elétrons se movem no campo estático dos íons. No caso de um cristal, os íons ocupam as posições definidas pela estrutura cristalina e o seguinte Hamiltoniano descreve a dinâmica eletrônica:

$$
\hat{H}=\sum_{i=1}^{N}\left(\frac{P_{i}^{2}}{2 m}+V_{l}\left(\vec{r}_{i}\right)\right)+\sum_{1 \leq i<j \leq N} V_{c}\left(\vec{r}_{i}-\vec{r}_{j}\right),
$$

onde $N$ é o número de elétrons, $V_{l}(\vec{r})$ é o potencial periódico da rede de íons e

$$
V_{c}(\vec{r})=\frac{e^{2}}{|\vec{r}|}
$$

é a repulsão Coulombiana entre os elétrons.

Apesar de simplificado pela aproximação de Born-Oppenheimer, o Hamiltoniano (3.1.1) 
ainda é complexo demais para ser diagonalizado. Uma primeira tentativa de simplificá-lo consiste em somar um potencial auxiliar $V_{A}(\vec{r})$ ao primeiro termo à direita e subtrair o mesmo potencial auxiliar no termo de dois corpos. Assim teremos que

$$
\hat{H}=\sum_{i=1}^{N}\left(\frac{P_{i}^{2}}{2 m}+V\left(\vec{r}_{i}\right)\right)+\sum_{1 \leq i<j \leq N} U\left(\vec{r}_{i}-\vec{r}_{j}\right),
$$

onde introduzimos os potenciais de um corpo e de dois corpos, $V(\vec{r})$ e $U\left(\vec{r}, \overrightarrow{r^{\prime}}\right)$, respectivamente:

$$
V(\vec{r})=V_{l}(\vec{r})+V_{A}\left(\overrightarrow{r^{\prime}}\right)
$$

e

$$
U\left(\vec{r}, \overrightarrow{r^{\prime}}\right)=V_{c}\left(\vec{r}-\overrightarrow{r^{\prime}}\right)-\left(\frac{1}{N-1}\right)\left(V_{A}(\vec{r})+V_{A}\left(\overrightarrow{r^{\prime}}\right)\right)
$$

O próximo passo é reescrever o Hamiltoniano em segunda quantização, numa base adequada. A construção da base começa com os autoestados da primeira soma no lado direito da equação (3.1.1), que define um Hamiltoniano $\hat{h}$, de partículas independentes. Como $V(\vec{r})$ é periódico, as autofunções $\varphi$ de $\hat{h}$ obedecem ao teorema de Bloch:

$$
\varphi_{\alpha, \vec{k}}(\vec{r})=e^{i \vec{k} \cdot \vec{r}} u_{\alpha, \vec{k}}(\vec{r})
$$

Aqui $u_{\alpha, \vec{k}}(\vec{r})$ tem a periodicidade da rede, o vetor de onda $\vec{k}$ é definido na primeira zona de Brillouin e o índice $\alpha$ indica a banda de energia a que pertence o autovalor correspondente. Temos então que

$$
\hat{h} \varphi_{\alpha, \vec{k}}(\vec{r})=\epsilon_{\alpha, \vec{k}} \varphi_{\alpha, \vec{k}}(\vec{r}) .
$$

As funções $\varphi_{\alpha, \vec{k}}$ constituem uma base em que o Hamiltoniano poderia ser projetado. Dado o termo de interação, porém, é mais conveniente definir uma nova base, de funções de Wannier $\phi_{\alpha}\left(\vec{r}-\vec{R}_{j}\right)$, onde $\vec{R}_{j}(j=1, \ldots, L)$ é um vetor da rede, onde $L$ é o número de íons. A função de Wannier $\phi_{\alpha}(\vec{r})$ é definida pela igualdade

$$
\phi_{\alpha}(\vec{r}) \equiv \frac{1}{\sqrt{L}} \sum_{\vec{k}} \varphi_{\alpha, \vec{k}}(\vec{r})
$$


Reciprocamente, as funções de Bloch podem ser obtidas das de Wannier:

$$
\varphi_{\alpha, \vec{k}}(\vec{r})=\frac{1}{\sqrt{L}} \sum_{j} e^{i \vec{k} \cdot \vec{R}_{j}} \phi_{\alpha}\left(\vec{r}-\vec{R}_{i}\right) .
$$

Definimos então o operador de Fermi $c_{\alpha, \vec{k}, \mu}^{\dagger}$, que cria um elétron com componente de spin $\mu$ no estado $\varphi_{\alpha, \vec{k}}(\vec{r})$. O operador

$$
c_{\alpha, j, a}^{\dagger}=\frac{1}{\sqrt{L}} \sum_{\vec{k}} e^{-i \vec{k} \cdot \vec{R}_{j}} c_{\alpha, \vec{k}, a}^{\dagger}
$$

cria um elétron com spin $\mu$ no estado de Wannier centrado em $\vec{R}_{j}$.

A partir dos $c_{\alpha, j, a}$ podemos definir os operadores de campo

$$
\Psi_{\mu}^{\dagger}(\vec{r})=\sum_{\alpha, j} \phi_{\alpha}^{*}\left(\vec{r}-\vec{R}_{j}\right) c_{\alpha, j, \mu}^{\dagger}
$$

os quais permitem reescrever o Hamiltoniano (3.1.1) na forma padrão

$$
\hat{H}=\sum_{\mu=\downarrow, \uparrow} \int \Psi_{\mu}^{\dagger}(\vec{r}) \hat{h} \Psi_{\mu} d r^{3}+\frac{1}{2} \sum_{\mu, \nu=\downarrow, \uparrow} \int \Psi_{\mu}^{\dagger}(\vec{r}) \Psi_{\nu}^{\dagger}\left(\overrightarrow{r^{\prime}}\right) U\left(\vec{r}, \overrightarrow{r^{\prime}}\right) \Psi_{\nu}\left(\overrightarrow{r^{\prime}}\right) \Psi_{\mu}(\vec{r}) d r^{3} d r^{\prime 3},
$$

a partir da qual a equação (3.1.11) conduz a uma expressão para o Hamiltoniano na base dos orbitais de Wannier:

$$
\hat{H}=\sum_{\alpha, i, j, \mu} t_{i, j}^{\alpha} c_{\alpha, i, \mu}^{\dagger} c_{\alpha, j, \mu}+\sum_{\alpha, \beta, \gamma, \delta, i, j, k, l} \sum_{\mu, \nu} U_{i, j, k, l}^{\alpha, \beta, \gamma} c_{\alpha, i, \mu}^{\dagger} c_{\beta, j, \nu}^{\dagger} c_{\gamma, k, \nu} c_{\delta, l, \mu} .
$$

Os elementos de matriz $t_{i, j}^{\alpha}$ são dados por

$$
t_{i, j}^{\alpha}=\int \phi_{\alpha}^{*}\left(\vec{r}-\vec{R}_{i}\right) \hat{h} \phi_{\alpha}\left(\vec{r}-\vec{R}_{j}\right)
$$

ou seja,

$$
t_{i, j}^{\alpha}=\frac{1}{L} \sum_{\vec{k}} e^{i \vec{k} \cdot\left(\vec{R}_{i}-\vec{R}_{j}\right)} \epsilon_{\alpha, \vec{k}}
$$

Analogamente calculamos os elementos de matriz $U_{i, j, k, l}^{\alpha, \beta, \gamma}$ :

$$
U_{i, j, k, l}^{\alpha, \beta, \gamma, \delta}=\int \phi_{\alpha}^{*}\left(\vec{r}-\vec{R}_{i}\right) \phi_{\beta}^{*}\left(\vec{r}-\vec{R}_{j}\right) U\left(\vec{r}, \overrightarrow{r^{\prime}}\right) \phi_{\gamma}\left(\vec{r}-\vec{R}_{k}\right) \phi_{\delta}\left(\vec{r}-\vec{R}_{l}\right) \mathrm{d} r^{3} \mathrm{~d} r^{\prime 3}
$$

A integral na equação (3.1.16) depende da superposição entre orbitais de Wannier, que 
podem ou não estar centrados no mesmo sítio ou pertencer à mesma banda. O modelo de Hubbard é obtido de (3.1.16) quando se admite que:

(i) os orbitais têm pequeno alcance, de forma que a superposição entre orbitais em sítios vizinhos é pequena e;

(ii) o nível de Fermi reside no interior de uma única banda $\alpha$ e fica bem abaixo das demais bandas de condução e bem acima das demais bandas de valência.

O Hamiltoniano pode então ser projetado na banda $\alpha$ e resulta que

$$
\hat{H}=\sum_{i, j, a} t_{i, j} c_{i, a}^{\dagger} c_{j, a}+U \sum_{i} c_{i, \uparrow}^{\dagger} c_{i, \downarrow}^{\dagger} c_{i, \downarrow} c_{i, \uparrow}
$$

Dentro do mesmo espírito, restringimos os termos definidos pelas equações (3.1.14) ou (3.1.15) a orbitais centrados em sítios vizinhos na estrutura cristalina, isto é, adotamos a aproximação de tight binding. Para abreviar, introduzimos os operadores número $n_{j, \uparrow}=c_{j, \uparrow}^{\dagger} c_{j, \uparrow}$ e $n_{j, \downarrow}=c_{j, \downarrow}^{\dagger} c_{j, \downarrow}$. Com isso, o Hamiltoniano (3.1.17) assume a forma

$$
\hat{H}=-t \sum_{\langle i, j\rangle} \sum_{\mu=\uparrow, \downarrow} c_{i, \mu}^{\dagger} c_{j, \mu}+U \sum_{j} n_{j, \uparrow} n_{j, \downarrow}
$$

onde o símbolo $\langle i, j\rangle$ denota uma soma restrita a pares de primeiros vizinhos.

Nesta dissertação, estamos interessados no modelo unidimensional (1-D) e trataremos exclusivamente dele daqui por diante. Em uma dimensão, o Hamiltoniano (3.1.17) pode ser escrito na forma mais simples

$$
\hat{H}=-t \sum_{j=1}^{L} \sum_{\mu=\uparrow, \downarrow}\left(c_{j, \mu}^{\dagger} c_{j+1, \mu}+H . c .\right)+U \sum_{j=1}^{L} n_{j, \uparrow} n_{j, \downarrow},
$$

onde $L$ é o número de sítios.

O primeiro termo à direita da equação (3.1.19) é a energia cinética. O segundo representa a interação entre elétrons, ou seja, a energia Coulombiana.

Podemos também escrever o Hamiltoniano de Hubbard num ensemble grã-canônico. Para isso, introduzimos o potencial químico $\mu$ e escrevemos que

$$
\hat{H}=-t \sum_{j=1}^{L} \sum_{\mu=\uparrow, \downarrow}\left(c_{j, \mu}^{\dagger} c_{j+1, \mu}+H . c .\right)+U \sum_{j=1}^{L} n_{j, \uparrow} n_{j, \downarrow}-\mu \sum_{j=1}^{L} n_{j} \text {, }
$$

onde $n_{j}=n_{j, \uparrow}+n_{j, \downarrow}$. 


\subsection{Exemplos paradigmáticos}

O Hamiltoniano (3.1.20) pode ser facilmente diagonalizado em dois extremos:

\subsubsection{Regime de interação forte}

Com $t=0$, não há hopping entre sítios. Basta considerar um sítio; cada nível assim encontrado será $L$ vezes degenerado. O Hamiltoniano que descreve um sítio tem a forma

$$
H_{1}=U n_{\uparrow} n_{\downarrow}-\mu n
$$

Podemos constituir uma base completa com quatro estados: $|\phi\rangle, c_{\uparrow}^{\dagger}|\phi\rangle, c_{\downarrow}^{\dagger}|\phi\rangle$ e $c_{\uparrow}^{\dagger} c_{\downarrow}^{\dagger}|\phi\rangle$, que são todos auto-estados de $H_{1}$, com energias $0,-\mu$, $-\mu$ e $U-2 \mu$, respectivamente. Se admitirmos que $U>\mu$, veremos que o estado fundamental é duplamente degenerado, com energia $-\mu$.

O estado fundamental do sistema de $L$ sítios será portanto $2^{L}$ vezes degenerado, com número de elétron $N=L$ e energia $E_{L}$ dada por $E_{L}-\mu N=-L \mu$, ou seja, $E_{L}=0$. No estado fundamental, cada sítio tem ocupação $n_{j}=1$, com spin $S_{z}= \pm 1 / 2$. A energia necessária para se adicionar um elétron será maior do que zero, porque com $N=L+1$ elétrons um dos sítios deverá ter ocupação dupla e energia $\epsilon_{j}$ tal que $\epsilon_{j}-2 \mu=U-2 \mu$, ou $\epsilon_{j}=U$. Os demais terão energia zero, e assim $E_{L+1}=U$.

A energia necessária $\mu_{+}$para adicionar um elétron será portanto

$$
\mu_{+} \equiv E_{L+1}-E_{L}=U
$$

Consideremos agora a energia para retirar um elétron. No estado fundamental com $N=$ $L-1$ elétrons, um dos sítios estará desocupado, mas sua energia será $\epsilon_{j}=0$, como a dos outros. Por isso, $E_{L-1}=0$ e a energia necessária para extrair uma partícula é

$$
\mu_{-} \equiv E_{L-1}-E_{L}=0
$$

O modelo de Hubbard 1-D com $t=0$ e $U>\mu$ tem portanto um gap de energia $\Delta \mu \equiv$ 
$\mu_{+}-\mu_{-}$dado por

$$
\Delta \mu=U
$$

\subsubsection{Regime de elétrons livres}

Com $U=0$, o Hamiltoniano de Hubbard se reduz a

$$
\hat{H}=-t \sum_{j} \sum_{\mu=\uparrow, \downarrow}\left(c_{j+1, \mu}^{\dagger} c_{j, \mu}+H . c .\right)-\mu N
$$

e é diagonalizado pela transformação

$$
c_{j, \sigma}=\frac{1}{\sqrt{N}} \sum_{k} e^{i k j} c_{k, \sigma} .
$$

A substituição dos operadores na equação (3.2.5) pelos operadores no lado direito da equação (3.2.6) conduz imediatamente ao resultado

$$
\hat{H}=-\frac{t}{N} \sum_{k, k^{\prime}, \sigma} c_{k, \sigma}^{\dagger} c_{k^{\prime}, \sigma} \sum_{j}\left(e^{i k j} e^{-i k^{\prime}(j+1)}+e^{i k j} e^{-i k^{\prime}(j-1)}\right)-\mu N,
$$

que pode ser reescrito na forma

$$
H=-t \sum_{k, k^{\prime}, \sigma} c_{k, \sigma}^{\dagger} c_{k^{\prime}, \sigma}\left(e^{-i k^{\prime}}+e^{i k^{\prime}}\right) \frac{1}{N} \sum_{j} e^{i\left(k-k^{\prime}\right) j}-\mu N .
$$

Uma vez que a última soma à direita na equação (3.2.8) se anula para $k^{\prime} \neq k$ e é unitária para $k^{\prime}=k$, chegamos à expressão diagonal

$$
\hat{H}=-2 t \sum_{k, \sigma} \cos (k) c_{k, \sigma}^{\dagger} c_{k, \sigma}-\mu N
$$

onde os momenta $k$ são definidos pela condição de contorno periódica:

$$
k=\frac{2 \pi \ell}{L} \quad(\ell=0,1,2, \ldots, L-1)
$$

A equação (3.2.9) define as energias de partícula independente

$$
\epsilon_{k}=-2 t \cos (k)-\mu \text {. }
$$

A figura (3.1) mostra a relação de dispersão na equação (3.2.11) para $\mu=0$. Para outros 
potenciais químicos, as curvas seriam deslocadas para cima $(\mu<0)$ ou para baixo $(\mu>0)$. Para qualquer potencial químico tal que $|\mu|<2 t$, o nível de Fermi $\epsilon_{F}=\mu$ estará no interior da banda, não haverá gap no nível de Fermi, e o sistema descrito pelo modelo será condutor. Essas características contrastam com a do regime de interação forte, descrito na sub-seção anterior, em que encontramos um gap, e o sistema era isolante $(N=L)$.

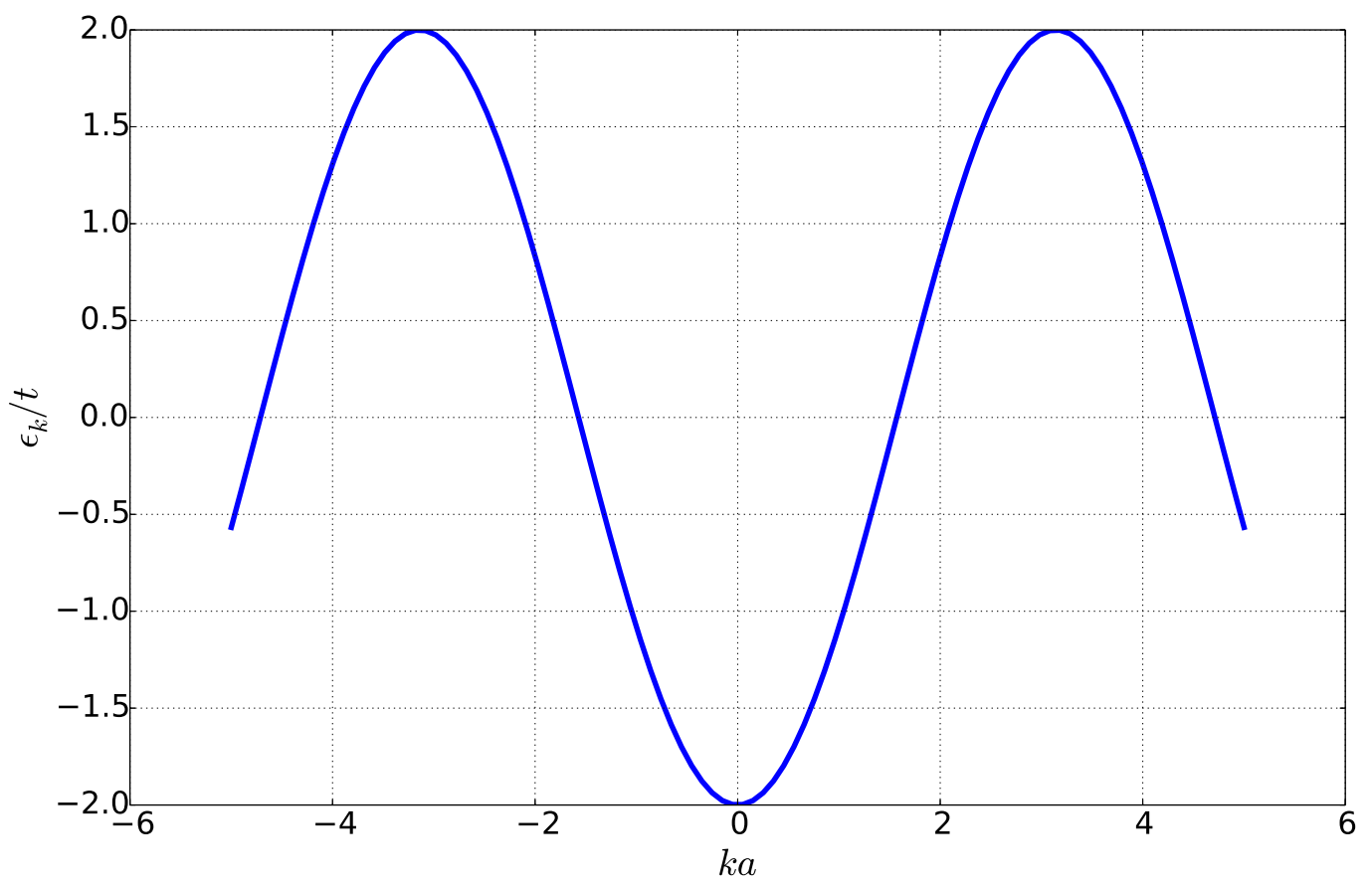

Figura 3.1 - Relação de dispersão para o modelo de Hubbard unidimensional sem interação $(U=0)$ e potencial químico $\mu=0$. No estado fundamental, os níveis abaixo da energia zero estão duplamente ocupados e os acima de zero estão vazios. As energias de partícula independente definem uma banda típica de condutores, com nível de Fermi no interior da banda. A constante da rede $a$ é igual a unidade e a constante de acoplamento $t$ também.

Fonte: Elaborada pelo autor. 
À medida que a relação $U / t$ cresce, portanto, o sistema deve evoluir de condutor para isolante. Veremos na seção (3.4) que ele se torna isolante assim que o coeficiente $U$ se torna positivo. O modelo de Hubbard 1-D sofre uma transição de Mott, a transição de fase discutida por Nevill Francis Mott (1905-1996) em que a interação eletrônica promove a passagem de um regime metálico para outro isolante, um fenômeno encontrado experimentalmente em materiais fortemente correlacionados. (13)

\subsection{As simetrias do modelo de Hubbard}

O Hamiltoniano de Hubbard possui simetrias que afetam marcadamente suas propriedades. Dentre elas destacaremos duas.

\subsubsection{Invariância translacional}

O Hamiltoniano (3.1.20) é invariante por translações ao longo da rede. De fato, uma translação equivale a mudança no índice mudo $j$ em cada uma das somas, o que não tem consequência prática. Pelo teorema de Noether, isso significa que o momentum eletrônico é conservado.

\subsubsection{Simetria partícula-buraco}

Uma transformação partícula-buraco leva um Hamiltoniano de Hubbard em outro Hamiltoniano de Hubbard que apenas difere do primeiro pelo potencial químico. Em particular, quando $U=2 \mu$, o Hamiltoniano permanece invariante.

Define-se a transformação partícula-buraco pela mudança nos operadores fermiônicos

$$
c_{j, \sigma}=(-1)^{j} a_{j, \sigma}^{\dagger} \quad(j=1, \ldots, L) .
$$

É fácil verificar que, sob essa transformação, o operador número de elétrons $n_{j, \sigma}$ se traduz 
em um operador número de buracos:

$$
c_{j, \sigma}^{\dagger} c_{j, \sigma}=a_{j, \sigma} a_{j, \sigma}^{\dagger}
$$

isto é,

$$
c_{j, \sigma}^{\dagger} c_{j, \sigma}=1-a_{j, \sigma}^{\dagger} a_{j, \sigma} .
$$

Aplicando as equações (3.3.1) e (3.3.3) no Hamiltoniano (3.1.20) e explorando as relações de comutação entre os operadores de Fermi $a_{j, \sigma}$, vemos que

$$
\hat{H}=-t \sum_{j, \sigma}\left(a_{j, \sigma}^{\dagger} a_{j+1, \sigma}+H . c .\right)+U \sum_{j=1}^{L}\left(1-a_{j, \uparrow}^{\dagger} a_{j, \uparrow}\right)\left(1-a_{j, \downarrow}^{\dagger} a_{j, \downarrow}\right)-\mu \sum_{j=1}^{L} \sum_{\sigma} a_{j, \sigma} a_{j, \sigma}^{\dagger} .
$$

Os dois últimos termos à direita na equação (3.3.4) podem ser simplificados e conduzem ao resultado

$$
\hat{H}=-t \sum_{j, \sigma}\left(a_{j, \sigma}^{\dagger} a_{j+1, \sigma}+H . c .\right)+U \sum_{j=1}^{2 L} \bar{n}_{j, \uparrow} \bar{n}_{j, \downarrow}-(U-\mu) \bar{N}+(U-2 \mu) L,
$$

onde

$$
\bar{n}_{j, \sigma} \equiv a_{j, \sigma}^{\dagger} a_{j, \sigma} \quad(\sigma=\uparrow, \downarrow)
$$

e

$$
\bar{N} \equiv \sum_{j, \sigma} \bar{n}_{j, \sigma}=2 L-N
$$

Na equação (3.3.5), o último termo à direita é uma constante que pode ser ignorada. A comparação dessa igualdade com a equação (3.1.20) mostra então que a transformação partícula-buraco leva o potencial químico $\mu$ em $U-\mu$, e o número de elétrons $N$ no número de buracos $\bar{N}=2 L-N$, mas não tem outro efeito sobre o Hamiltoniano. O Hamiltoniano simétrico, definido pela igualdade $U=2 \mu$ e $N=L$, é invariante sob a transformação, já que nesse caso o potencial químico não muda e $\bar{N}$ coincide com $N$, o que implica em uma banda semi-preenchida.

Um caso particular interessante é $N=L-1$, isto é, banda quase semi preenchida. Nesse caso o potencial químico é igual a $\mu_{-}$, a energia necessária para retirar um elétron de uma 
banda semi-preenchida

$$
\mu=\mu_{-}=E(L, U)-E(L-1, U) .
$$

A transformação partícula-buraco nos conduz então a

$$
\bar{N}=L+1
$$

e

$$
\mu=\mu_{+}=E(L+1, U)-E(L, U) .
$$

Onde $\mu_{+}$é a energia para adicionar um elétron a uma banda semi-preenchida. Temos então que

$$
\mu_{+}=U-\mu_{-}
$$

ou seja,

$$
\mu_{+}+\mu_{-}=U
$$

No capítulo 4, teremos oportunidade de explorar a equação (3.3.12). A invariância sob transformação partícula-buraco será também muito valiosa, porque permitirá considerar apenas números de elétrons satisfazendo à condição $N \leq L$. Se o número de partículas for superior a $L$, efetuaremos uma transformação partícula-buraco para mapear o Hamiltoniano em outro com $\bar{N}<L$.

O modelo de Hubbard é exatamente solúvel, via ansatz de Bethe, como discutido a seguir.

\subsection{A solução pelo ansatz de Bethe}

Em 1931 Hans Bethe (1906-2005) propôs um procedimento para diagonalizar exatamente o modelo de Heisenberg unidimensional com spin-1/2. (14) Em 1938 Lamek Hulthén (19091995) desenvolveu o chamado ansatz (do alemão "palpite educado") de Bethe para o modelo antiferromagnético de Heisenberg e encontrou o estado fundamental em uma rede infinitamente longa. Desde então o ansatz de Bethe se consolidou como uma ferramenta poderosa na teoria da matéria condensada. 
O ansatz consiste em propor que, no espaço das posições, a função de onda em cada região do espaço é uma superposição de ondas planas. Mais especificamente, escreve-se a função de onda na forma

$$
|\Psi\rangle=\sum_{1 \leq x_{1}<\ldots<x_{n} \leq N} \sum_{P \in S_{N}} A_{P} e^{\sum_{j=1}^{n} i k_{P_{j}} x_{j}}\left|x_{1}, \ldots, x_{n}\right\rangle,
$$

onde $N$ é o número de elétrons e $P_{j}$ é uma permutação dos índices dentro do conjunto $S_{N}$ de todas as permutações compatíveis com o número de elétrons, com coeficientes $A_{P}$ escolhidos para diagonalizar o Hamiltoniano.

Essa condição sobre os coeficientes $A_{P}$ resulta nas equações de Bethe. A Ref. (9) encontra as equações de Bethe para o modelo de Hubbard 1-D, do que resultam expressões para duas densidades $\rho$ e $\sigma$, associadas à carga e ao spin, respectivamente:

$$
2 \pi \rho(k)=1+\cos (k) \int_{-B}^{B} \frac{8 U \sigma(\Lambda)}{U^{2}+16(\sin (k)-\Lambda)^{2}} d \Lambda
$$

e

$$
\int_{-Q}^{Q} \frac{8 U \rho(k)}{U^{2}+16(\Lambda-\sin (k))^{2}} d k=2 \pi \sigma(\Lambda)+\int_{-B}^{B} \frac{4 U \sigma(\bar{\Lambda})}{U^{2}+4(\Lambda-\bar{\Lambda})^{2}} d \bar{\Lambda} .
$$

Aqui os $k$ são momenta e os $\Lambda$ também são números reais. Os limites $Q$ e $B$ são determinados pelo número de elétrons e pela magnetização do sistema:

$$
\int_{-Q}^{Q} \rho(k) d k=\frac{N}{L}
$$

e

$$
\int_{-B}^{B} \sigma(\Lambda) d \Lambda=\frac{M}{L}
$$

onde $N$ é o número de elétrons e $M$ é o número de elétrons com spin $\downarrow$.

A solução das equações (3.4.2)-(3.4.5) determina a densidade de momenta e assim permite determinar a energia, dada por uma equação formalmente idêntica à expressão para a energia do gás de elétrons livres discutido na sub-seção sobre o regime de elétrons livres:

$$
E=-2 \sum_{j=1}^{N} \cos \left(k_{j}\right)
$$


que assim assume a forma

$$
E=-2 L \int_{-Q}^{Q} \rho(k) \cos (k) d k .
$$

A expressão $M / L$ cresce monotonicamente conforme $B$ cresce, alcançando o máximo $(1 / 2)$ quando $B \rightarrow \infty$. Analogamente temos que $N / L$ atinge o máximo (1) quando $Q=\pi$.

No caso $B \rightarrow \infty$ e $Q=\pi$ as equações (3.4.2)-(3.4.5) admitem solução analítica e pode mostrar-se que a energia do estado fundamental é dada pela expressão exata

$$
E=-4 L \int_{0}^{\infty} \frac{\mathrm{J}_{0}(x) \mathrm{J}_{1}(x)}{x\left(1+e^{x U / 2}\right)} d x
$$

onde $\mathrm{J}_{0}(x)$ e $\mathrm{J}_{1}(x)$ são as funções de Bessel de ordem 0 e ordem 1 , respectivamente.

A equação (3.4.5) permite calcular a energia para $N=L$ e qualquer $U$. A Ref. (11) propôs uma parametrização que é exata para qualquer $N \leq L$ quando $U=0$ ou $U \rightarrow \infty$ e serve como aproximação entre esses extremos:

$$
e^{B A}(n, U)=-\frac{2 \beta(U)}{\pi} \sin \left(\frac{n \pi}{\beta(U)}\right) \quad(n \leq 1),
$$

onde $n=N / L$ e $\beta(U)$ é dado pela seguinte expressão:

$$
-\frac{2 \beta(U)}{\pi} \sin \left(\frac{\pi}{\beta(U)}\right)=-4 \int_{0}^{\infty} \frac{\mathrm{J}_{0}(x) \mathrm{J}_{1}(x)}{x\left(1+e^{\frac{U x}{2}}\right)} d x .
$$

Dada uma intensidade de interação $U$, a equação (3.4.10) permite encontrar o parâmetro $\beta$. A partir dele, a equação (3.4.9) determina a energia do estado fundamental para qualquer densidade $n \leq 1$. Para densidades no intervalo $1<n \leq 2$, a transformação partícula-buraco gera uma aproximação equivalente:

$$
e^{B A}(n, U)=-\frac{2 \beta(U)}{\pi} \sin \left(\frac{(2-n) \pi}{\beta(U)}\right) \quad(1 \leq n \leq 2)+U(n-1) .
$$

Com isso, tem-se em mãos expressões que determinam, aproximadamente, a energia do estado fundamental no sistema homogêneo. A partir delas, a maquinaria da DFT permite encontrar a energia e a densidade dos estados fundamentais de sistemas não-homogêneos. 


\section{Resultados}

A teoria do funcional da densidade é formalmente exata. No entanto, como explicado no Capítulo 2, a precisão dos cálculos depende, na prática, da aproximação para o funcional de troca e correlação $E_{x c}[n]$ e da aproximação para o potencial de troca e correlação $V_{x c}[n]$. Quanto melhor a aproximação, melhores os resultados. O potencial $V_{x c}[n]$ é comumente calculado por meio de uma derivada funcional, como indicado pela Eq. (2.2.8). Assim, procura-se uma aproximação para o funcional $E_{x c}[n]$, a partir da qual por derivação se encontra o potencial $V_{x c}[n]$.

Neste capítulo, que toma o modelo de Hubbard 1-D como campo de prova, trataremos das duas aproximações. No que tange ao potencial, mostraremos que uma LDA mais precisa é obtida diretamente do potencial químico para o sistema uniforme. No que diz respeito a $E_{x c}[n]$, proporemos um funcional híbrido, mais preciso que o BA-LDA. Em ambos os casos, trabalharemos com base na conexão adiabática, ainda que, no caso do potencial de troca e correlação, pudéssemos chegar às mesmas conclusões diretamente da construção de Kohn e Sham.

\subsection{A conexão adiabática no modelo de Hubbard}

Como vimos no Capítulo 3, em uma dimensão o modelo de Hubbard pode ser escrito como

$$
\hat{H}=-t \sum_{j}\left(c_{j}^{\dagger} c_{j+1}+H . c .\right)+\sum_{j} v_{j} \hat{n}_{j}+U \sum_{j} \hat{n}_{j \uparrow} \hat{n}_{j \downarrow}-\sum_{j} \mu \hat{n}_{j} .
$$

Queremos expressar o terceiro termo no lado direito da equação acima, que define a interação Coulombiana $\hat{W}$, em termos do operador densidade total $\hat{n}=\hat{n}_{\uparrow}+\hat{n}_{\downarrow}$. Para isso adotamos a proposta da Ref. (11) para o termo de interação e assim temos que

$$
\hat{H}=-t \sum_{j}\left(c_{j}^{\dagger} c_{j+1}+H . c .\right)+\sum_{j} v_{j} n_{j}+\frac{U}{4} \sum_{j} \hat{n}_{j}^{2}-\mu N
$$


A derivação da expressão (2.3.12), que expressa a conexão adiabática, pode agora ser estendida, sem nenhuma modificação, para o modelo de Hubbard 1-D. Temos então que

$$
E[n]=\left\langle\psi_{0}\left|\hat{T}_{s}\right| \psi_{0}\right\rangle+\left\langle\psi_{0}|\hat{V}| \psi_{0}\right\rangle+\int_{0}^{1}\left\langle\psi_{\lambda}|\hat{W}| \psi_{\lambda}\right\rangle \mathrm{d} \lambda
$$

A energia do sistema de Kohn e Sham, que constitui o primeiro termo no lado direito na equação acima, pode ser escrito na forma

$$
T_{s}[n]=\sum_{\ell=1}^{N} \epsilon_{\ell}-\sum_{j} v_{j}^{s} n_{j} .
$$

Na primeira soma à direita as parcelas são as energias de Kohn-Sham $\epsilon_{\ell}$ abaixo do potencial químico $\mu_{s}$, do sistema não-interagente. Os $\epsilon_{\ell}$ são resultantes da diagonalização do Hamiltoniano não interagente

$$
\hat{H}_{s}=-t \sum_{j}\left(c_{j}^{\dagger} c_{j+1}+H . c .\right)+\sum_{j} v_{j}^{s} \hat{n}_{j}-\mu_{s} N
$$

onde $v^{s}$ é o potencial de Kohn-Sham:

$$
v_{j}^{s}=v_{j}+v_{H}[n]_{j}+v_{x c}[n]_{j} .
$$

Na equação acima, $v_{H}$ é o potencial de Hartree, que precisa ser derivado da energia de Hartree $E_{H}[n]$. Esta última é definida pela Ref. (11) como

$$
E_{H}[n]=\frac{U}{4} \sum_{j} n_{j}^{2} .
$$

Para obter o potencial de Hartree no sítio $j$ basta agora derivar da energia de Hartree, equação (4.1.8), em relação à densidade $n_{j}$ :

$$
v_{H}[n]_{j}=\frac{U}{2} n_{j}
$$

Com o auxílio das equações (4.1.5), (4.1.7) e (4.1.9) podemos escrever (4.1.4) como 


$$
E[n]=\sum_{\ell=1}^{N} \epsilon_{\ell}-\sum_{j}\left(v_{j}+\frac{U}{4} n_{j}+v_{x c}[n]_{j}\right) n_{j}+\sum_{j} v_{j} n_{j}+\int_{0}^{1}\left\langle\psi_{\lambda}|\hat{W}| \psi_{\lambda}\right\rangle \mathrm{d} \lambda
$$

ou após cancelamento dos termos devidos ao potencial $\hat{V}$,

$$
E[n]=\sum_{\ell=1}^{N} \epsilon_{\ell}-\sum_{j}\left(\frac{U}{4} n_{j}+v_{x c}[n]_{j}\right) n_{j}+\int_{0}^{1}\left\langle\psi_{\lambda}|\hat{W}| \psi_{\lambda}\right\rangle \mathrm{d} \lambda .
$$

\subsection{O potencial químico}

O modelo de Hubbard unidimensional exibe gaps de energia, que se manifesta por uma descontinuidade no potencial químico $\mu$. O potencial químico de um sistema é definido como a energia necessária para adicionar um elétron. Normalmente, as energias para subtrair ou para adicionar um elétron só diferem porque têm sinais opostos. Em certos casos, porém, a energia $\mu_{+}$para adicionar um elétron é diferente de $\mu_{-}$, que é o negativo da energia necessária para subtrair uma partícula. Nesses casos, diz-se que o sistema tem um gap de energia $E_{\text {gap }}=\mu_{+}-\mu_{-}$.

Na DFT, o potencial químico tem uma interpretação bem conhecida. (15) Para discuti-la no modelo de Hubbard, vamos imaginar que o Hamiltoniano em consideração não possua gap e que a densidade eletrônica $n$ sofra uma variação $\delta n$ de tal forma que $\sum_{j} \delta n_{j}=1$, isto é, que $n+\delta n$ contenha exatamente um elétron a mais do que a densidade $n$ do estado fundamental de um Hamiltoniano de Hubbard. Como o sistema é infinito, a variação é infinitesimal.

A variação $\delta E$ da energia fundamental $E[n]$ devida a $\delta n$ pode ser decomposta em duas partes:

(i) A energia varia porque a densidade não é mais a densidade do estado fundamental;

(ii) A energia varia porque o sistema ganha um elétron.

Como a energia do estado fundamental é mínima, a variação (i) é necessariamente nula. Resta a parcela (ii), e assim.

$$
\delta E=\mu
$$


onde $\mu$ é o potencial químico. A energia $\delta E$ pode, por outro lado, ser extraída da equação (4.1.11):

$$
\delta E=\sum_{\ell=1}^{N+1} \epsilon_{\ell}-\sum_{\ell=1}^{N} \epsilon_{\ell}+\delta\left(-\sum_{j}\left(\frac{U}{4} n_{j}+v_{x c}[n]_{j}\right) n_{j}+\int_{0}^{1}\left\langle\psi_{\lambda}|\hat{W}| \psi_{\lambda}\right\rangle \mathrm{d} \lambda\right) .
$$

$\mathrm{Na}$ equação (4.2.2), empregamos o Teorema de Hellmann-Feynman para notar que os $\epsilon_{\ell}$ não são afetados pela variação $\delta n$. Entretanto, uma vez que a densidade $n+\delta n$ comporta uma partícula adicional, mais um nível de Kohn e Sham precisou ser preenchido. Já a variação no terceiro termo à direita é nula, porque ela descreve a variação da energia do estado fundamental sob uma variação infinitesimal da densidade. Vemos assim que

$$
\delta E=\epsilon_{N+1}
$$

assim da equação (4.2.1) temos

$$
\mu=\epsilon_{N+1} .
$$

O potencial químico é, portanto, o $N+1$-ésimo autovalor de Kohn e Sham. Uma vez que inexiste gap, esse autovalor difere infinitesimalmente do $N$-ésimo autovalor, e assim, a equação acima equivale a afirmar que

$$
\mu=\epsilon_{N}
$$

Poderíamos ter chegado diretamente à mesma conclusão se tivéssemos escolhido $\delta n$ de forma que $\sum_{j} \delta n_{j}=-1$.

\subsection{O potencial de troca e correlação}

Dada uma aproximação para o funcional de troca e correlação $E_{x c}[n]$, o potencial de troca e correlação sempre pode ser computado por derivação funcional. Como aproximação para o funcional do modelo de Hubbard 1-D, a Ref. (11) propôs uma aproximação de densidade local com base na solução pelo ansatz de Bethe (BA-LDA) descrita na última seção do capítulo 3. 
A partir da equação (3.4.9), a seguinte expressão de LDA pode ser escrita para a energia do estado fundamental:

$$
E[n]=-\frac{2 \beta(U)}{\pi} \sum_{j} \begin{cases}\operatorname{sen}\left(\frac{\pi n_{j}}{\beta(U)}\right) & \left(0 \leq n_{j} \leq 1\right) \\ \operatorname{sen}\left(\frac{\pi\left(2-n_{j}\right)}{\beta(U)}\right)+U\left(n_{j}-1\right) & \left(1 \leq n_{j} \leq 2\right)\end{cases}
$$

Para obter a BA-LDA, devemos agora considerar a expressão de Kohn e Sham que defne o funcional de troca e correlação no caso de potencial externo nulo:

$$
E_{x c}[n]=E[n]-T_{s}[n]-E_{H}[n] .
$$

A equação acima mostra que precisamos apenas conhecer a energia cinética $T_{s}[n]$ e a energia de Hartree $E_{H}[n]$ para obter, aproximadamente, $E_{x c}$.

A energia cinética pode ser calculada como soma das energias abaixo da energia de Fermi do gás 1-D de elétrons livres. Alternativamente, ela pode ser obtida da própria equação (3.4.9), que se torna exata no limite $U \rightarrow 0$. Da equação (3.4.10) e da igualdade

$$
\int_{0}^{\infty} \frac{\mathrm{J}_{0}(x) \mathrm{J}_{1}(x)}{x} d x=\frac{2}{\pi}
$$

temos imediatamente que

$$
\beta(U=0)=2,
$$

e assim obtemos o resultado

$$
T_{s}[n]=-\frac{4}{\pi} L \sin (n \pi / 2)
$$

onde $L$ é o número de sítios da rede. Podemos agora combinar as equações acima para gerar uma expressão aproximada para o funcional de troca e correlação:

$$
E_{x c}[n]=\sum_{j} e_{x c}\left[n_{j}\right]
$$

onde 
$e_{x c}\left[n_{j}\right]=\frac{4}{\pi} \operatorname{sen}\left(\frac{\pi n_{j}}{2}\right)-\frac{U}{4} n_{j}^{2}-\frac{2 \beta(U)}{\pi} \begin{cases}\operatorname{sen}\left(\frac{\pi n_{j}}{\beta(U)}\right) & \left(0 \leq n_{j} \leq 1\right) \\ \operatorname{sen}\left(\frac{\pi\left(2-n_{j}\right)}{\beta(U)}\right)+U\left(n_{j}-1\right) & \left(1 \leq n_{j} \leq 2\right) .\end{cases}$

Onde o parâmetro $\beta(U)$ é obtido a partir da solução da equação (3.4.10). Como ele independe da densidade $n_{j}$, a igualdade é facilmente diferenciada em relação a $n_{j}$ para gerar a seguinte expressão para o potencial de troca e correlação:

$$
v_{x c}\left[n_{j}\right]=2 \operatorname{sen}\left(\frac{\pi n_{j}}{2}\right)-\frac{U}{2} n_{j}-2 \begin{cases}\cos \left(\frac{\pi n_{j}}{\beta(U)}\right) & \left(0 \leq n_{j} \leq 1\right) \\ -\cos \left(\frac{\pi\left(2-n_{j}\right)}{\beta(U)}\right)+U & \left(1 \leq n_{j} \leq 2\right) .\end{cases}
$$

A equação (4.3.7) envolve duas aproximações: A parametrização (3.4.9) das energias na solução pelo ansatz de Bethe e a LDA embutida na equação (4.3.2). A equação (4.3.8) depende das mesmas duas aproximações, mas gera desvios maiores, porque a derivada que conduz a ela amplia os erros erros embutidos na parametrização. Esclareçamos com um exemplo.

\subsubsection{O problema da derivada}

Na LDA, a energia de troca e correlação é uma função da densidade local $n_{j}$. Admitamos, para fins de ilustração, que o funcional exato fosse também uma função, a senóide $e_{x c}\left(n_{j}\right)=$ $\sin \left(n_{j}\right)$, mostrada em azul na Fig. 4.1, e que um cálculo aproximado conduzisse à função $\bar{e}_{x c}\left(n_{j}\right)=n_{j}\left(1-n_{j}^{2} / 2\right)$, mostrado em verde na mesma figura. 


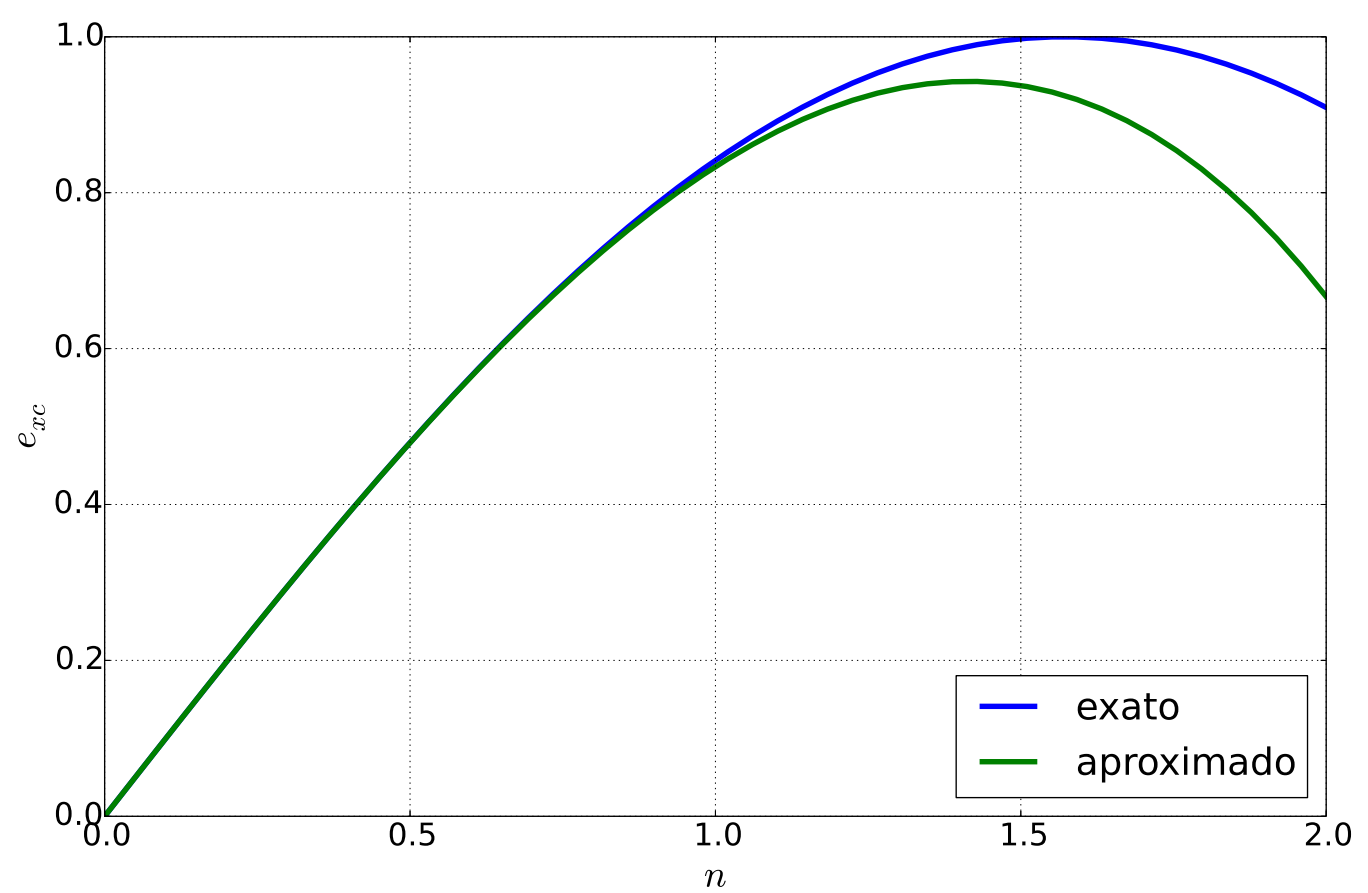

Figura 4.1 - Exemplo de energia de troca e correlação, uma curva para o valor exato (em azul) e outra para a aproximação (em verde).

Fonte: Elaborada pelo autor.

O gráfico mostra que as duas funções praticamente se confundem para $n_{j}<1$, mas se separam para $n_{j}>1$. A Fig. 4.2, entretanto, mostra que a derivada da função aproximada se afasta rapidamente da derivada da exata. Os desvios são grandes para $n_{j}>0.3$. 


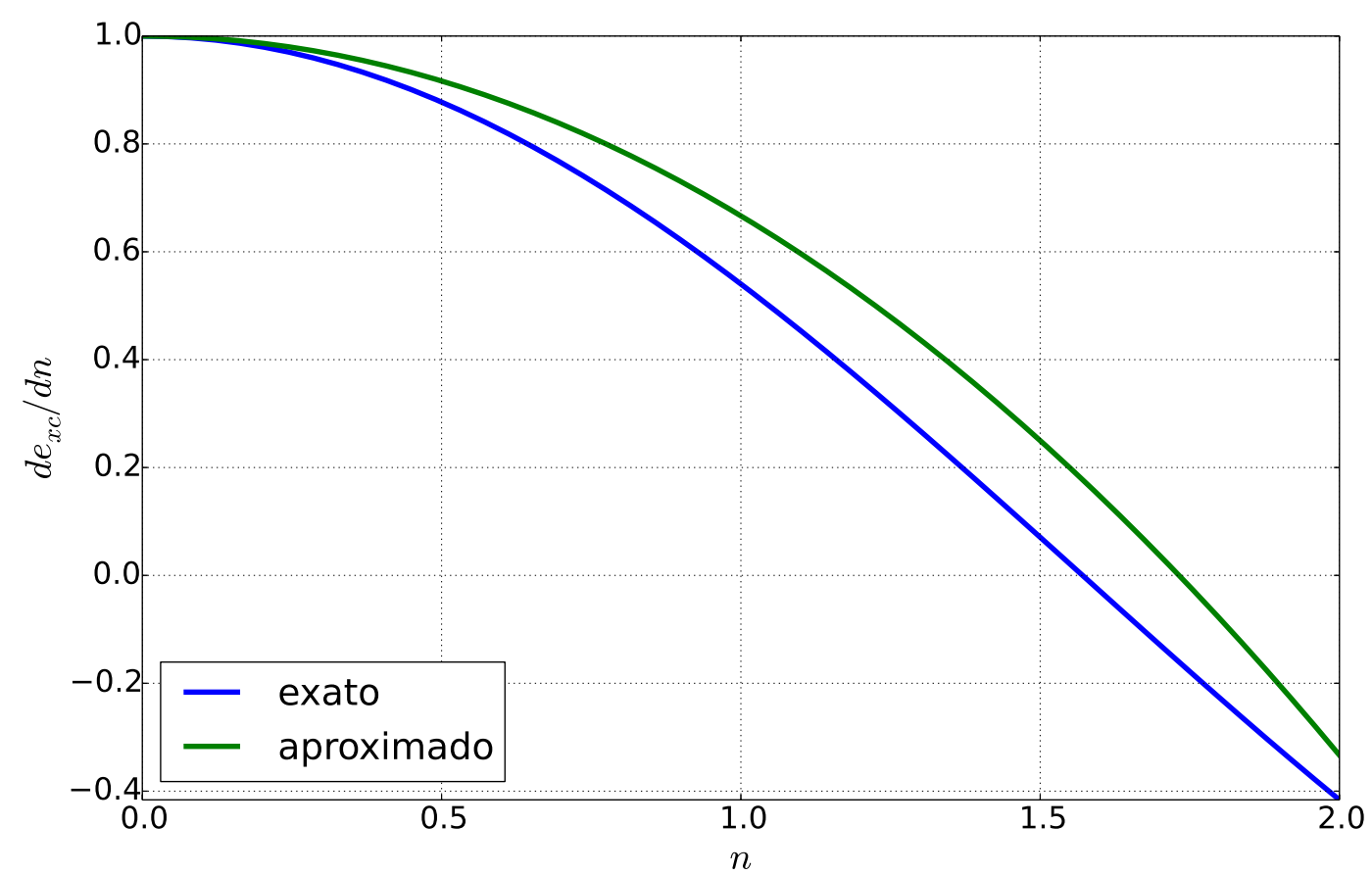

Figura 4.2 - Exemplo de potecial de troca e correlação, uma curva para o valor exato (em azul) e outra para a aproximação (em verde).

Fonte: Elaborada pelo autor.

A derivação amplifica os desvios porque a aproximação em $\bar{e}_{x c}$ se torna menos precisa à medida que $n_{j}$ cresce. A mesma limitação afeta a equação (4.3.7), que é exata para $n_{j}=0$, $n_{j}=1$ e $n_{j}=2$, mas não para valores intermediários de $n_{j}$. Por isso, é desejável evitar a diferenciação da expressão aproximada para o funcional de troca e correlação. A próxima seção explica como isso pode ser feito.

\subsection{Derivação alternativa do potencial de troca e cor- relação}

Queremos encontrar uma aproximação de densidade local para o potencial de troca e correlação. Para isso, queremos aplicar a equação $\mu=\epsilon_{N}$ ao Hamiltoniano uniforme, o que exige conhecimento dos autovalores de Kohn e Sham. No sistema uniforme, o potencial externo é nulo, e a equação (4.1.7), com a equação (4.1.9), toma a forma

$$
v^{s}[n]=\frac{n}{2} U+v_{x c}[n] .
$$


Uma vez que é uniforme, o potencial de Kohn e Sham apenas desloca os autovalores em relação aos de um sistema livre, que obedecem à relação de dispersão

$$
\epsilon_{\ell}^{0}=-2 t \cos k \quad k= \pm \frac{2 \pi \ell}{L}\left(\ell=0,1,2, \ldots, \frac{L}{2}\right) .
$$

Os autovalores de Kohn e Sham são portanto

$$
\epsilon_{\ell}=\left(\frac{n}{2}\right) U+v_{x c}[n]-2 t \cos k \quad k= \pm \frac{2 \pi \ell}{L}\left(\ell=0,1,2, \ldots, \frac{L}{2}\right) .
$$

Como o sistema contém $N$ elétrons, os $N / 2$ níveis de Kohn e Sham mais baixos são ocupados, isto é, $N / 4$ níveis com $\ell>0$ e $N / 4$ com $\ell<0$. Os níveis mais elevados têm portanto $\ell= \pm \bar{\ell} \equiv N / 4$ e energia

$$
\epsilon_{\bar{\ell}}=\left(\frac{n}{2}\right) U+v_{x c}[n]-2 t \cos \left(\frac{\pi n}{2}\right)
$$

De acordo com a equação (4.2.5), o lado direito da equação acima deve ser igual ao potencial químico, e assim chegamos a uma LDA para o potencial de troca e correlação:

$$
v_{x c}[n]=\mu+2 t \cos \left(\frac{\pi n}{2}\right)-\left(\frac{n}{2}\right) U
$$

Como argumentado, temos que o resultado acima é mais preciso que a derivação dada pela equação (2.2.8). Para analisar sua precisão aplicaremos as duas maneiras de calcular $v_{x c}[n]$ no modelo de Hubbard 1-D.

\subsection{O modelo de Hubbard 1-D}

No capítulo 3 vimos que o modelo de Hubbard possui um gap, mais precisamente, o modelo de Hubbard possui um gap na banda semi-preenchida $(n=1)$ quando $U>0$. Calculemos então $v_{x c}\left[n=1^{+}\right]-v_{x c}\left[n=1^{-}\right]$pela derivada, usando o ansatz de Bethe como sistema referencial, e pela nossa expressão (4.4.5).

\section{Pela derivada:}

Calculamos a derivada da energia na equação (4.3.8), basta apenas subtrair $v_{x c}\left[n=1^{+}\right]$ de $v_{x c}\left[n=1^{-}\right]$. Assim temos que 


$$
v_{x c}\left[n=1^{+}\right]-v_{x c}\left[n=1^{-}\right]=U+4 \cos (\pi / \beta(U)) .
$$

Pela conexão adiabática:

Pela expressão (4.4.5) temos que

$$
v_{x c}\left[n=1^{+}\right]-v_{x c}\left[n=1^{-}\right]=\mu_{+}-\mu_{-}
$$

Onde $\mu_{+}$e $\mu_{-}$foram definidos nas equações (3.3.8) e (3.3.10) e são calculados na Ref. $(9,16)$ Assim temos que

$$
\mu_{-}=2-2 \int_{0}^{\infty} \frac{\mathrm{J}_{1}(x) e^{x U / 4}}{x\left(1+e^{x U / 4}\right)} d x
$$

E temos também que a diferença entre $\mu_{+}$e $\mu_{-}$é

$$
\mu_{+}-\mu_{-}=-4+4 \int_{0}^{\infty} \frac{\mathrm{J}_{1}(x) e^{x U / 4}}{x\left(1+e^{x U / 4}\right)} d x+U
$$

Defina a função $\gamma(U)$ dada implicitamente pela seguinte equação:

$$
\cos \left(\frac{\pi}{\gamma(U)}\right)=2 \int_{0}^{\infty} \frac{\mathrm{J}_{1}(x)}{x\left(1+e^{x U / 2}\right)} d x-1+U .
$$

Usando a função $\gamma(U)$ podemos escrever

$$
v_{x c}\left[n=1^{+}\right]-v_{x c}\left[n=1^{-}\right]=U+4 \cos \left(\frac{\pi}{\gamma(U)}\right) .
$$

Assim podemos então comparar a equação (4.5.1), em azul, e a equação (4.5.6), em verde, no seguinte gráfico: 


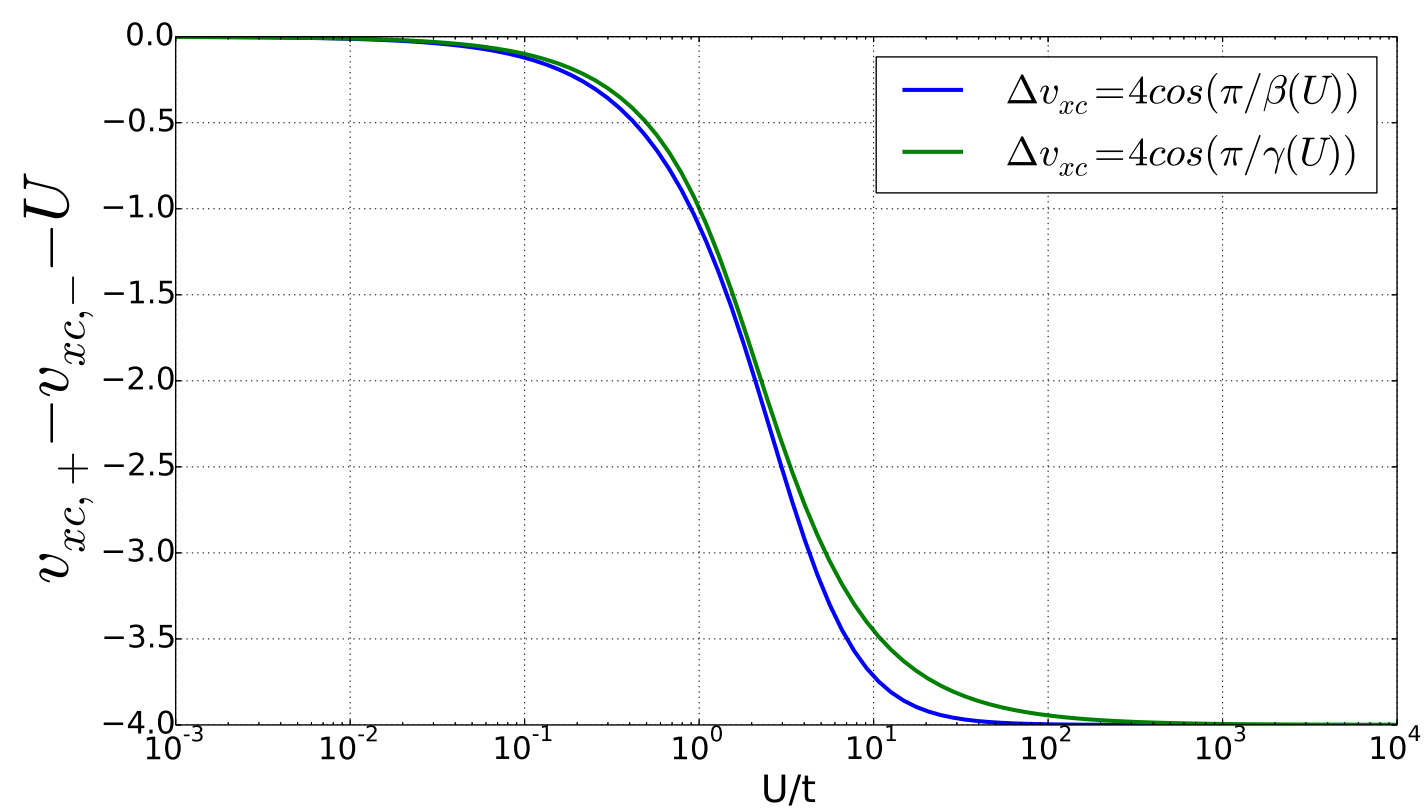

Figura 4.3 - No eixo da abscissa temos o parâmetro U, em escala logarítmica. A equação (4.5.1) a menos do $U$, advinda da derivação da energia, está em azul. Em verde está a equação (4.5.6), também subtraído $U$, advinda da conexão adiabática.

Fonte: Elaborada pelo autor.

Conforme demonstra a figura (4.3) a equação (4.5.6) difere da (4.5.1), principalmente no intervalo entre $10^{0}$ e $10^{2}$. Confirmando assim que a expressão sem derivada é mais precisa para o cálculo do valor do gap.

\subsection{Funcionais Híbridos}

Como discutido no primeiro capítulo, os funcionais híbridos geram aproximações não-locais para o funcional de troca e correlação. Para melhor entender a estrutura desse procedimento, convém começar com uma aproximação mais simples, inicialmente defendida por argumentos intuitivos. Suponhamos que as equações de Kohn e Sham, com a LDA para o funcional de troca e correlação, tenham sido resolvidas para um dado sistema físico. Da solução resultam várias informações: Os auto-estados e auto-valores de Kohn e Sham, a densidade e a energia do estado fundamental, entre outras. Tomando esses dados como base, é possível nesse ponto adotar-se um aproximação de Hartree-Fock.

Se o sistema for uma molécula, por exemplo, pode tomar-se o determinante de Slater constituído pelos orbitais de Kohn e Sham para construir uma função de onda do estado 
fundamental e, a partir dela, seguir a receita do método de Hartree-Fock para calcular o potencial de troca (não-local). Esse potencial $v_{x}[n]$ substituiria o potencial $v_{x c}[n]$ fornecido pela LDA. Por ser derivado do ciclo auto-consistente de Kohn e Sham, o potencial $v_{x}[n]$ depende apenas de $n$ e assim pode ser visto como uma aproximação para o potencial de troca e correlação.

Substituir o potencial $v_{x c}[n]$ da LDA por $v_{x}[n]$ tem uma vantagem e duas desvantagens. A vantagem é que o potencial $v_{x}[n]$ leva em conta efeitos não-locais que $v_{x c}[n]$ desconsidera. As desvantagens são que:

(i) é bem mais custoso trabalhar com determinantes de Slater do que com a densidade;

(ii) a aproximação de Hartree-Fock deixa de lado a correlação e assim se torna imprecisa quando a interação é pequena em comparação com a energia cinética.

Com relação ao custo, nada se pode fazer, mas a seguinte discussão mostra como a construção de funcionais híbridos reduz a outra desvantagem (falta de correlação) sem prejudicar a vantagem.

A construção é guiada pela conexão adiabática, equação (2.3.12). Podemos comparar a conexão adiabática com o funcional de troca e correlação $E_{x c}[n]$ definido na segunda seção do capítulo 2, vemos que a conexão adiabática oferece uma expressão explícita para o funcional de troca e correlação:

$$
E_{x c}[n]=\int_{0}^{1}\left\langle\psi_{\lambda}|\hat{W}| \psi_{\lambda}\right\rangle d \lambda-E_{H}[n] .
$$

Em linhas gerais, a equação acima mostra que, a menos do termo de Hartree, o funcional de troca e correlação é uma média entre os valores médios esperados da interação $\hat{W}$ no estado fundamental do sistema não-interagente de Kohn e Sham $(\lambda=0)$ e no estado fundamental do sistema interagente $(\lambda=1)$, conforme apontado pela Ref. (17), que consideraram um gás tridimensional de elétrons com interação Coulombiana. No modelo de Hubbard, a mesma ideia pode ser explorada mais a fundo e quantitativamente. Para isso, precisaremos obter a energia do estado fundamental na aproximação de Hartree-Fock para o modelo de Hubbard, o que faremos a seguir. 


\subsubsection{A aproximação de Hartree-Fock}

Para obter a aproximação de Hartree-Fock, voltamos ao Hamiltoniano de Hubbard e escrevemos em seguida os operadoress densidade no termo de interação como a densidade $\hat{n}_{j \mu}$ $(\mu=\uparrow, \downarrow)$ mais uma flutuação:

$$
\hat{n}_{j \mu}=n_{j \mu}+\left(\hat{n}_{j \mu}-n_{j \mu}\right) .
$$

A substituição no termo de interação nos dá então que

$$
\begin{array}{r}
\hat{H}=-t \sum_{j}\left(c_{j}^{\dagger} c_{j+1}+H . c .\right)+\sum_{j} v_{j} n_{j}+U \sum_{j}\left(\left(\hat{n}_{j \uparrow}-n_{j \uparrow}\right)\left(\hat{n}_{j \downarrow}-n_{j \downarrow}\right)\right. \\
\left.+\left(\hat{n}_{j \uparrow}-n_{j \uparrow}\right) n_{j \downarrow}+\left(\hat{n}_{j \downarrow}-n_{j \downarrow}\right) n_{j \uparrow}+n_{j \uparrow} n_{j \downarrow}\right) .
\end{array}
$$

O primeiro termo dentro da parcela de interação no lado direito, proporcional ao quadrado da flutuação, é pequeno e será ignorado. Resta então o resultado

$$
\hat{H}_{H}=-t \sum_{j}\left(c_{j}^{\dagger} c_{j+1}+H . c .\right)+\sum_{j} v_{j} n_{j}+U \sum_{j}\left(\hat{n}_{j \uparrow} n_{j \downarrow}+\hat{n}_{j \downarrow} n_{j \uparrow}-n_{j \uparrow} n_{j \downarrow}\right) .
$$

De posse dessa expressão, podemos agora escrever uma expressão para o valor esperado da energia de troca, isto é, do termo da interação no Hamiltoniano de Hartree-Fock, no estado fundamental:

$$
\left\langle\psi_{0}\left|\hat{W}_{H F}\right| \psi_{0}\right\rangle=U \sum_{j} n_{j \uparrow} n_{j \downarrow}
$$

Para obter a contribuição da troca para o funcional da energia do estado fundamental precisamos apenas subtrair a energia de Hartree $E_{H}[n]=U n^{2} / 4$ do lado direito:

$$
E_{x}^{H F}=U \sum_{j}\left(n_{j \uparrow} n_{j \downarrow}-\frac{1}{2} n_{j}^{2}\right)
$$

Em um sistema não polarizado, $n_{j \mu}=n / 2(\mu=\uparrow, \downarrow)$, a equação acima se reduz a 


$$
E_{x}^{H F}=-\frac{U}{4} \sum_{j} n_{j}^{2} .
$$

\subsubsection{O funcional híbrido para o modelo de Hubbard 1-D}

Podemos agora derivar uma expressão de funcional híbrido para o Hamiltoniano de Hubbard. Para isso, antes de mais nada, precisamos modificar o lado direito da equação (4.6.1). Uma vez que a energia de Hartree independe de $\lambda$, temos que

$$
E_{x c}[n]=\int_{0}^{1}\left\langle\psi_{\lambda}|\hat{W}| \psi_{\lambda}\right\rangle-E_{H}[n] d \lambda
$$

e podemos focalizar a atenção no integrando do lado direito.

O valor médio esperado no integrando pode ser obtido da BA-LDA. Sabemos do capítulo 3 que a energia média por partícula para um sistema com coeficiente de interação $U \lambda$ é dada pela igualdade

$$
e^{B A}(n, \lambda U)=-\frac{2}{\pi} \beta \operatorname{sen}\left(\frac{n \pi}{\beta}\right),
$$

onde o parâmetro $\beta=\beta(U \lambda)$ é dado pela solução da equação

$$
\beta \operatorname{sen}\left(\frac{\pi}{\beta}\right)=2 \pi \int_{0}^{\infty} \frac{J_{0}(x) J_{1}(x)}{x\left(1+e^{\lambda U x / 2}\right)} \mathrm{d} x
$$

Uma vez que o valor médio $\left\langle\psi_{\lambda}|\hat{W}| \psi_{\lambda}\right\rangle$ pode ser calculado com base no teorema de Hellmann-Feynman,

$$
\left\langle\psi_{\lambda}|\hat{W}| \psi_{\lambda}\right\rangle=\frac{d E}{d \lambda}
$$

convém derivar a equação (4.6.9) em relação a $\lambda$ :

$$
\frac{\partial e^{B A}}{\partial \lambda}=-\frac{2}{\pi}\left(\operatorname{sen}\left(\frac{n \pi}{\beta}\right)-\frac{n \pi}{\beta} \cos \left(\frac{n \pi}{\beta}\right)\right) \frac{d \beta}{d \lambda} .
$$

O último fator à direita na equação (4.6.11) pode ser obtido da equação (4.6.10). Diferenciamos os dois lados para ver que 


$$
\left(\sin (\pi / \beta)-\frac{\pi}{\beta} \cos (\pi / \beta)\right) \frac{d \beta}{d \lambda}=U \pi \int_{0}^{\infty} \frac{\mathrm{J}_{0}(x) \mathrm{J}_{1}(x) e^{U x \lambda / 2}}{\left(1+e^{U x \lambda / 2}\right)^{2}} d x
$$

e assim a equação (4.6.12) se escreve na forma

$$
\frac{\partial e^{B A}}{\partial \lambda}=-2 U \frac{\sin \left(\frac{n \pi}{\beta}\right)-\frac{n \pi}{\beta} \cos \left(\frac{n \pi}{\beta}\right)}{\sin \left(\frac{\pi}{\beta}\right)-\frac{\pi}{\beta} \cos \left(\frac{\pi}{\beta}\right)} \int_{0}^{\infty} \frac{\mathrm{J}_{0}(x) \mathrm{J}_{1}(x) e^{U x \lambda / 2}}{\left(1+e^{U x \lambda / 2}\right)^{2}} d x
$$

Combinando a equação acima com a equação (4.6.11) para ver que

$$
\left\langle\psi_{\lambda}|\hat{W}| \psi_{\lambda}\right\rangle=-2 U \int_{0}^{\infty} \frac{\mathrm{J}_{0}(x) \mathrm{J}_{1}(x) e^{U x \lambda / 2}}{\left(1+e^{U x \lambda / 2}\right)^{2}} d x \sum_{j} \frac{\sin \left(\frac{n_{j} \pi}{\beta}\right)-\frac{n_{j} \pi}{\beta} \cos \left(\frac{n_{j} \pi}{\beta}\right)}{\sin \left(\frac{\pi}{\beta}\right)-\frac{\pi}{\beta} \cos \left(\frac{\pi}{\beta}\right)}
$$

A integral presente no lado direito da equação (4.6.14) independe da densidade. No intervalo $0 \leq n \leq 1$, somando no último termo à direita é unitário e máximo quando $n_{j}=1$ e se anula quando $n_{j}=0$. Para sermos específicos, consideraremos a densidade unitária na seguinte discussão; resultados análogos seriam obtidos para outras densidades. Com $n_{j}=1$, temos que

$$
\left\langle\psi_{\lambda}|\hat{W}| \psi_{\lambda}\right\rangle=-2 L U \int_{0}^{\infty} \frac{\mathrm{J}_{0}(x) \mathrm{J}_{1}(x) e^{U x \lambda / 2}}{\left(1+e^{U x \lambda / 2}\right)^{2}} d x
$$

ou se preferirmos considerar o integrando no lado direito da equação (4.6.8), temos assim que

$$
\left\langle\psi_{\lambda}|\hat{W}| \psi_{\lambda}\right\rangle-E_{H}=U L\left(-2 \int_{0}^{\infty} \frac{\mathrm{J}_{0}(x) \mathrm{J}_{1}(x) e^{U x \lambda / 2}}{\left(1+e^{U x \lambda / 2}\right)^{2}} d x-\frac{1}{4}\right)
$$




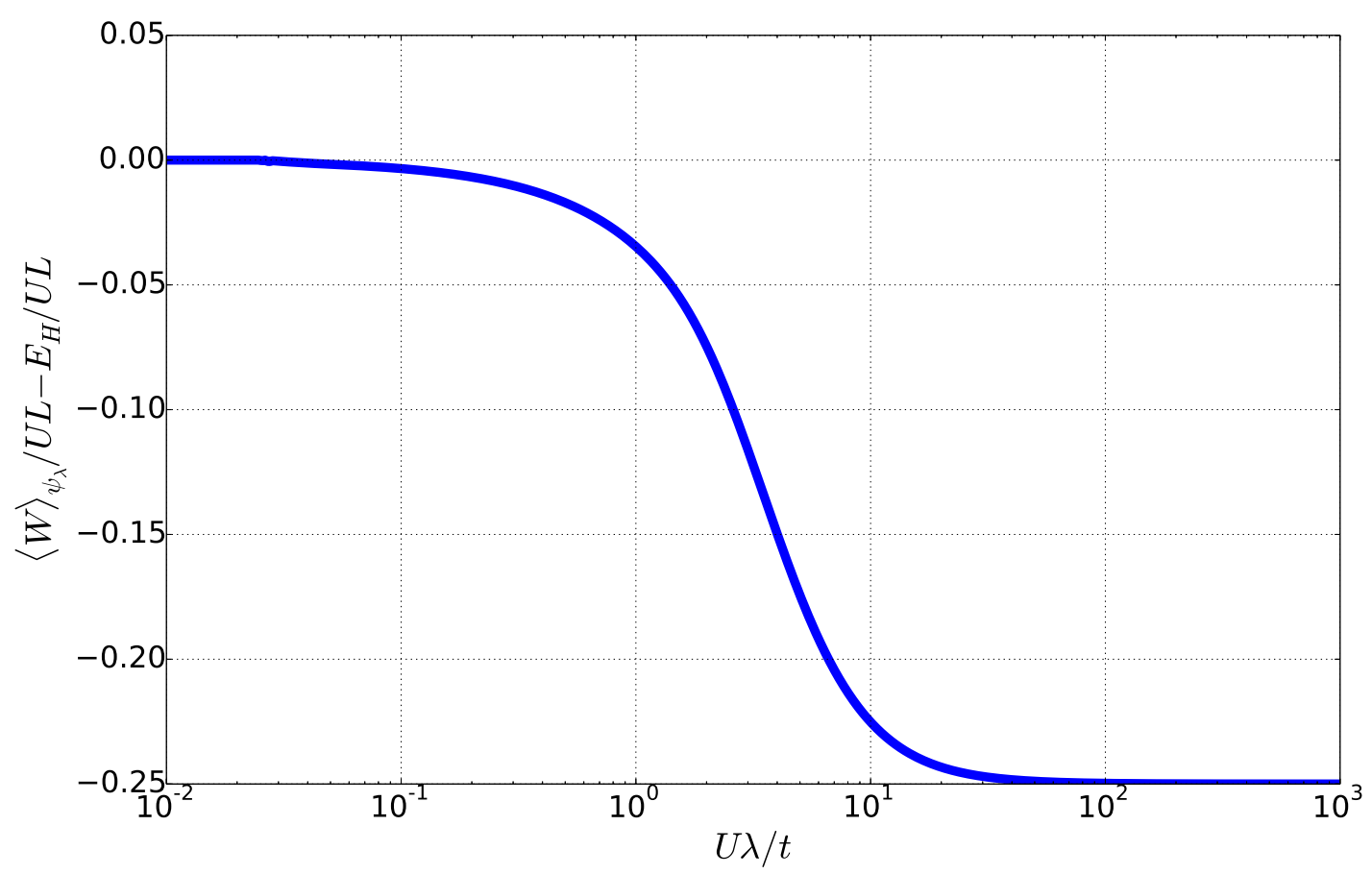

Figura 4.4 - Valor médio esperado da energia de interação $\hat{W}$ no estado fundamental do Hamiltoniano de Hubbard unidimensional para densidade eletrônica unitária, medido a partir da energia de Hartree, em função da coeficiente de Coulomb $U$ e dividido pelo número de sítios $L$, para densidade eletrônica unitária $(n=1)$. A ordenada é expressa em unidades de $U$ vezes o número de sítios, enquanto a abscissa é expressa em unidades do acoplamento $t$.

Fonte: Elaborada pelo autor.

O gráfico acima mostra o termo entre parênteses na equação (4.6.17) em função de $U \lambda / t$. A partir dele podemos discutir o valor da energia de troca e correlação dado pela equação (4.6.8) dividida pelo número de sítios $L$. Dado um coeficiente de interação $U$, a integral à direita na equação (4.6.17) corresponde a integrar a função na Fig. (4.4) entre zero e o valor escolhido para $U$ e multiplicar o resultado por $U$ vezes o número de sítios.

Assim, para $U \lambda<0.1 t$, região em que a curva da figura fica muito perto de zero, o valor de $e_{x c}[n]$ será muito pequeno, onde $e[n]$ é a energia por número de sítios $L$. Estamos nesse caso no regime de troca, em que a correlação é muito pequena e a energia de troca e correlação é bem descrita pela teoria de Hartree-Fock.

À medida que $U$ se torna maior, a partir de $U \lambda=0.1 t$, a correlação se torna progressivamente mais importante e esperamos que a aproximação de Hartree-Fock deixe de ser precisa. Nessa região, precisaremos recorrer à LDA para descrever $e_{x c}[n]$. Mais precisamente, para $U \lambda>0.1 t$, é necessário quebrar a integral no lado direito da equação (4.6.8) em duas partes e escrever 


$$
e_{x c}[n]=\int_{0}^{t / 10 U}\left\langle\psi_{\lambda}|\hat{W}| \psi_{\lambda}\right\rangle-e_{H} d \lambda+\int_{t / 10 U}^{1}\left\langle\psi_{\lambda}|\hat{W}| \psi_{\lambda}\right\rangle-e_{H} d \lambda
$$

No primeiro termo, o integrando pode ser substituído pela expressão de Hartree-Fock (4.6.7) para a energia de troca, que independe de $\lambda$. Assim obtemos a seguinte igualdade, que define o funcional híbrido de troca e correlação para o modelo de Hubbard 1-D:

$$
e_{x c}[n]=\left\{\begin{array}{l}
-\frac{U}{8} n^{2} \quad(U \leq 0.1 t) \\
-\frac{t}{80} n^{2}+e_{x c}^{L D A}[n]-e_{x c, \lambda=t / 10 U}^{L D A} \quad(U>0.1 t),
\end{array}\right.
$$

onde $e_{x c}^{L D A}[n]$ é uma aproximação de densidade local para o funcional da energia de troca e correlação por número de sítios, a BA-LDA, por exemplo. E $e_{x c, \lambda=t / 10 U}^{L D A}[n]$ é o funcional calculado em $\lambda=\frac{t}{10 U}$. 


\section{Considerações Finais}

Sabendo da dificuldade da LDA em repoduzir gaps, (18) como o do modelo de Hubbard 1-D, aplicamos a BA-LDA (equação (4.5.1)) e o potencial químico (equação (4.5.6)) para calcular o gap do potencial químico. De fato as duas expressões reproduzem um gap, porém a nossa expressão (4.5.6), advinda da conexão adiabática, proporciona um resultado mais preciso que o obtido pela BA-LDA, já que independe de derivadas.

Funcionais híbridos são construídos a partir da conexão adiabática. (17) Assim usando nossa aplicação da conexão adiabática no modelo de Hubbard 1-D obtivemos um importante resultado, a figura (4.4). Com o gráfico em mãos, e com o auxílio da aproximação de HartreeFock, pudemos escrever a expressão (4.6.19), que merece ser relembrada:

$$
e_{x c}[n]=\left\{\begin{array}{l}
-\frac{U}{8} n^{2} \quad(U \leq 0.1 t) \\
-\frac{t}{80} n^{2}+e_{x c}^{L D A}[n]-e_{x c, \lambda=t / 10 U}^{L D A} \quad(U>0.1 t) .
\end{array}\right.
$$

Esse é o nosso funcional híbrido para a energia de troca e correlação do modelo de Hubbard unidimensional. Uma possível continuação deste trabalho seria aplicar os resultados aqui obtidos a vários sistemas unidimensionais, para testes de precisão e custo computacional. 


\section{REFERÊNCIAS}

1 HOHENBERG, P.; KOHN, W. Inhomogeneous electron gas. Physical Review, v. 136, n. 3B, p. B864-B871, 1964.

2 KOHN, W.; SHAM, L. J. Self-consistent equations including exchange and correlation effects. Physical Review, v. 140, n. 4A, p. A1133-A1138, 1965.

3 CAPELLE, K. A bird's-eye view of density-functional theory. Brazilian Journal of Physics, v. 36, n. 4 A, p. 1318-1343, 2006.

4 JONES, R. O. Density functional theory: its origins, rise to prominence, and future. Reviews of Modern Physics, v. 87, n. 3, p. 897-923, 2015.

5 GUNNARSSON, O.; HJELMBERG, H. Hydrogen chemisorption by the spin-density functional formalism. i. Physica Scripta, v. 11, n. 2, p. 97, 1975.

6 GUNNARSSON, O.; JOHANSSON, P.; LUNDQVIST, S.; LUNDQVIST, B. I. Chemical binding in small molecules by the spin-density-functional formalism. International Journal of Quantum Chemistry, v. 9, n. S9, p. 83-93, 1975.

7 MUSCAT, J.; WANDER, A.; HARRISON, N. On the prediction of band gaps from hybrid functional theory. Chemical Physics Letters, v. 342, n. 3-4, p. 397-401, 2001.

8 BECKE, A. D. A new mixing of hartree-fock and local density-functional theories. Journal of Chemical Physics, v. 98, n. 2, p. 1372-1377, 1993.

9 LIEB, E. H.; WU, F. Y. Absence of mott transition in an exact solution of the short-range, one-band model in one dimension. Physical Review Letters, v. 20, n. 25, p. 1445-1448, 1968.

10 FERMI, E. Eine statistische methode zur bestimmung einiger eigenschaften des atoms und ihre anwendung auf die theorie des periodischen systems der elemente. Zeitschrift für Physik, v. 48, n. 1, p. 73-79, 1928.

11 LIMA, N. A.; et al. Density functionals not based on the electron gas: local-density approximation for a luttinger liquid. Physical Review Letters, v. 90, n. 14, p. 146402, 2003.

12 GUNNARSSON, O.; LUNDQVIST, B. I. Exchange and correlation in atoms, molecules, and solids by the spin-density-functional formalism. Physical Review B, v. 13, n. 10, p. 4274-4298, 1976. 
13 MOTT, N. F. The basis of the electron theory of metals, with special reference to the transition metals. Proceedings of the Physical Society A, v. 62, n. 7, p. 416, 1949.

14 BETHE, H. Zur theorie der metalle. Zeitschrift für Physik, v. 71, n. 3, p. 205-226, 1931.

15 LUNDQVIST, S.; MARCH, N. H. Theory of the inhomogeneous electron gas. New York: Springer, 1983.

16 ESSLER, H. L. E.; et al. The one-dimensional hubbard model. Cambridge: Cambridge University Press, 2005.

17 PERDEW, J. P.; ERNZERHOF, M.; BURKE, K. Rationale for mixing exact exchange with density functional approximations. Journal of Chemical Physics, v. 105, n. 22, p. 9982-9985, 1996.

18 PERDEW, J. P. Density functional theory and the band gap problem. International Journal of Quantum Chemistry, v. 28, n. S19, p. 497-523, 1985. 